\title{
TOPOLOGICAL DERIVATIVES FOR SEMILINEAR ELLIPTIC EQUATIONS
}

\author{
Mohamed IGUERNANE* *,SERguei A. NAZAROV **, JEAN-Rodolphe ROCHE*, \\ JAN SOKOLOWSKI* KATARZYNA SZULC * \\ * Laboratoire de Mathématiques, Institut Elie Cartan \\ Université Henri Poincaré, UMR 7502 Nancy-Université-CNRS-INRIA \\ Nancy 1, B.P. 239, 54506 Vandoeuvre lès Nancy Cedex, France \\ e-mail: \{Mohamed. Iguernane, Jean-Rodolphe.Roche\} @iecn.u-nancy.fr \\ \{Jan.Sokolowski, Katarzyna.Szulc\} @iecn.u-nancy.fr \\ ** Institute of Mechanical Engineering Problems \\ V.O., Bolshoi pr., 61, 199178, St. Petersburg, Russia \\ e-mail: serna@snark.ipme.ru, srgnazarov@yahoo.co.uk
}

\begin{abstract}
The form of topological derivatives for an integral shape functional is derived for a class of semilinear elliptic equations. The convergence of finite element approximation for the topological derivatives is shown and the error estimates in the $L^{\infty}$ norm are obtained. The results of numerical experiments which confirm the theoretical convergence rate are presented.
\end{abstract}

Keywords: shape optimization, topological derivative, levelset method, variational inequality, asymptotic analysis.

\section{Introduction}

1.1. Topological derivatives in shape optimization. Topological derivatives are introduced for linear problems in (Sokolowski and Zochowski, 1999) and for variational inequalities in (Sokolowski and Zochowski, 2005). The mathematical theory of asymptotic analysis is applied in (Nazarov and Sokolowski, 2003; 2006) for the derivation of topological derivatives in shape optimization of elliptic boundary values problems. Numerical solutions of shape optimization problems for variational inequalities obtained by the level set method combined with topological derivatives are presented in (Fulmanski et al., 2007)

In the paper we present topological derivatives for semilinear elliptic boundary value problems. In the first part, asymptotic analysis of a class of boundary value problems for a second order semilinear differential equation is performed. In the second part, the convergence of our finite element approximation for the topological derivatives is proved, and the results of numerical experiments are presented as well.

Topological sensitivity analysis aims to provide an asymptotic expansion of a shape functional with respect to the size of a small hole created inside the domain. For a criterion $j(\Omega)=\mathcal{J}\left(u_{\Omega} ; \Omega\right)$, where $\Omega \subset \mathbb{R}^{N}(N=2$ or 3) and $u_{\Omega}$ is a solution of a set of partial differential equations defined over $\Omega$, this expansion can be generally written in the form

$$
j\left(\Omega \backslash\left(\overline{\mathcal{O}+\omega_{\varepsilon}}\right)\right)-j(\Omega)=f(\varepsilon) \mathcal{T}_{\Omega}(\mathcal{O}, \omega)+o(f(\varepsilon)) .
$$

Here $\varepsilon$ and $\mathcal{O}$ denote respectively the diameter and the center of the hole, $\omega$ is a fixed domain containing the origin $\mathcal{O}$ and $f(\varepsilon)$ is a positive function tending to zero with $\varepsilon$. The coefficient $\mathcal{T}_{\Omega}$ is commonly called the topological derivative.

1.2. Semilinear elliptic equation. Let $\Omega$ and $\omega$ be bounded domains in $\mathbb{R}^{3}$ with the smooth boundaries $\partial \Omega$ and $\partial \omega$ and the compact closures $\bar{\Omega}$ and $\bar{\omega}$, respectively. The origin $\mathcal{O}$ of the coordinate system is assumed to belong to the domains $\Omega$ and $\omega$. The following sets are introduced:

$$
\begin{aligned}
\omega_{\varepsilon} & =\left\{x \in \mathbb{R}^{3}: \xi:=\varepsilon^{-1} x \in \omega\right\}, \\
\Omega(\varepsilon) & :=\Omega \backslash \overline{\omega_{\varepsilon}},
\end{aligned}
$$

where $x=\left(x_{1}, x_{2}, x_{3}\right)$ are Cartesian coordinates in the domain $\Omega$ and $\varepsilon>0$ is a small parameter. The upper bound $\varepsilon_{0}>0$ is chosen in such a way that for $\varepsilon \in\left(0, \varepsilon_{0}\right]$ the set $\overline{\omega_{\varepsilon}}$ belongs to the domain $\Omega$. We can diminish the value of $\varepsilon_{0}>0$ in the sequel, if necessary. However, the 
notation for the bound $\varepsilon_{0}$ remains unchanged. The set $\omega_{\varepsilon}$ is called a hole, or an opening, in the domain $\Omega(\varepsilon)$.

In this paper, we consider a nonlinear elliptic problem in the singularly perturbed domain $\Omega(\varepsilon)$ :

$$
\left\{\begin{aligned}
-\Delta_{x} u^{\varepsilon}(x) & =F\left(x, u^{\varepsilon}(x)\right), & & x \in \Omega(\varepsilon), \\
u^{\varepsilon}(x) & =0, & & x \in \partial \Omega(\varepsilon) .
\end{aligned}\right.
$$

Here $F \in C^{0, \alpha}(\Omega \times \mathbb{R})$ and $f \in C^{0, \alpha}(\Omega)$ are given functions, independent of the parameter $\varepsilon$. Asymptotic analysis in the linear case is well known (see the monographs (Il'in, 1989; Mazja et al., 1991)), e.g., for the Dirichlet boundary value problem for the Poisson equation:

$$
\left\{\begin{aligned}
-\Delta_{x} u^{\varepsilon}(x) & =f(x), & & x \in \Omega(\varepsilon), \\
u^{\varepsilon}(x) & =0, & & x \in \partial \Omega(\varepsilon) .
\end{aligned}\right.
$$

According to the method of compound asymptotic expansions (Mazja et al., 1991), in asymptotic analysis of (4) there appear two limit problems. The first one is obtained by formally taking $\varepsilon=0$, e.g., by filling the hole $\overline{\omega_{\varepsilon}}$ :

$$
\left\{\begin{aligned}
-\Delta_{x} u(x) & =f(x), & & x \in \Omega, \\
u(x) & =0, & & x \in \partial \Omega,
\end{aligned}\right.
$$

and the second one is the boundary value problem, which furnishes the leading boundary layers term:

$$
\left\{\begin{aligned}
-\Delta_{\xi} w(\xi) & =0, & & \xi \in \mathbb{R}^{3} \backslash \bar{\omega} \\
w(\xi) & =-u(\mathcal{O}), & & \xi \in \partial \omega
\end{aligned}\right.
$$

where $u(\mathcal{O})$ is the value at the origin of the solution of (5).

As in (Mazja et al., 1981) (see also Ch. 5.7 in (Mazja et al., 1991)), for the nonlinear problem (3) we obtain also two limit problems. The first one is nonlinear,

$$
\left\{\begin{aligned}
-\Delta_{x} v(x) & =F(x, v(x)), & & x \in \Omega, \\
v(x) & =0, & & x \in \partial \Omega,
\end{aligned}\right.
$$

and the second one is the linear exterior problem (6) with $u(\mathcal{O}):=v(\mathcal{O})$ given by the solution to $(7)$.

Our aim in this paper is the construction of asymptotic approximations for solutions to (3) in such a way that we will be able to obtain an expansion of a given shape functional

$$
\mathcal{J}\left(u^{\varepsilon} ; \Omega(\varepsilon)\right)=\int_{\Omega(\varepsilon)} J\left(x, u^{\varepsilon}(x)\right) \mathrm{d} x,
$$

of the first order with respect to $\varepsilon$, namely,

$$
\mathcal{J}\left(u^{\varepsilon} ; \Omega(\varepsilon)\right)=\mathcal{J}(v ; \Omega)+\varepsilon \mathcal{T}_{\Omega}(\mathcal{O})+o(\varepsilon),
$$

(cf. (1)), where

$$
\mathcal{J}(v ; \Omega)=\int_{\Omega} J(x, v(x)) \mathrm{d} x,
$$

and $\mathcal{T}_{\Omega}$ is the topological derivative of the functional $\mathcal{J}$.

Apart from that, we need the linearized problem (7), which gives us the regular terms in the asymptotic approximation,

$$
\left\{\begin{aligned}
-\Delta_{x} V(x)-F_{v}^{\prime}(x, v(x)) V(x) & =\mathcal{F}(x), & & x \in \Omega, \\
V(x) & =g(x), & & x \in \partial \Omega .
\end{aligned}\right.
$$

The solution $V$ is but the so-called the adjoint state. The adjoint state is introduced in order to simplify the expression for the topological derivative.

Appropriate function spaces are employed to analyze the solvability of all boundary value problems introduced above. The weighted Hölder spaces $\Lambda_{\beta}^{l, \alpha}(\Omega)$ are defined (Mazja and Plamenevskii, 1978) as the closure of $C_{c}^{\infty}(\bar{\Omega} \backslash$ $\mathcal{O})$ (smooth functions vanishing in the vicinity of $\mathcal{O}$ ) in the norm

$$
\begin{aligned}
& \left\|Z ; \Lambda_{\beta}^{l, \alpha}(\Omega)\right\| \\
& =\sum_{k=0}^{l} \sup _{x \in \Omega}|x|^{\beta-l-\alpha+k}\left|\nabla_{x}^{k} Z(x)\right| \\
& +\sup _{x, y \in \Omega,|x-y|<|x| / 2}|x|^{\beta}|x-y|^{-\alpha}\left|\nabla_{x}^{l} Z(x)-\nabla_{y}^{l} Z(y)\right| .
\end{aligned}
$$

The standard norm in the Hölder space $C^{l, \alpha}(\Omega)$ is as follows:

$$
\begin{aligned}
& \left\|Z ; C^{l, \alpha}(\Omega)\right\| \\
& =\sum_{k=0}^{l} \sup _{x \in \Omega}\left|\nabla_{x}^{k} Z(x)\right| \\
& \quad+\sup _{x, y \in \Omega,|x-y|<|x| / 2}|x-y|^{-\alpha}\left|\nabla_{x}^{l} Z(x)-\nabla_{y}^{l} Z(y)\right| .
\end{aligned}
$$

Here $l \in\{0,1, \ldots\}, \alpha \in(0,1)$ and $\beta \in \mathbb{R}$.

Now we introduce several assumptions which are required to define the topological derivatives:

(H1) The limit problem (7) has a solution $v \in C^{2, \alpha}(\Omega)$ and $F \in C^{0,1}(\bar{\Omega} \times \mathbb{R})$ with a certain $\alpha \in(0,1)$.

(H2) The linear problem (11) with $\mathcal{F} \in C^{0, \alpha}(\Omega), g \in$ $C^{2, \alpha}(\partial \Omega)$ has a unique solution $V \in C^{2, \alpha}(\Omega)$,

$$
\left\|V ; C^{2, \alpha}(\Omega)\right\| \leq c\left(\left\|\mathcal{F} ; C^{0, \alpha}(\Omega)\right\|+\left\|g ; C^{2, \alpha}(\partial \Omega)\right\|\right) .
$$

Here and in the sequel $c$ stands for a positive constant that may change from place to place but never depends on $\varepsilon$. (H3) $F_{v}^{\prime} \in C^{0, \alpha}(\bar{\Omega} \times \mathbb{R})$.

If (H3) holds true and $F_{v}^{\prime}(x, v(x)) \leq 0$ for $x \in \Omega$, then (H2) is also satisfied.

The hypothesis (H2) means the existence and uniqueness of classical solutions to the linearized problem in Hölder spaces $C^{2, \alpha}(\Omega)$ with the a priori estimate (12). It turns out that the linear mapping for the problem (11), i.e.,

$$
S:\{\mathcal{F}, g\} \longmapsto V,
$$


is an isomorphism in the Hölder spaces $C^{0, \alpha}(\Omega) \times$ $C^{2, \alpha}(\partial \Omega) \rightarrow C^{2, \alpha}(\Omega)$. By a general result in (Mazja and Plamenevskii, 1978), (see also (Nazarov and Plamenevsky, 1994)), the operator remains to be an isomorphism in weighted Hölder spaces under the proper choice of indices.

Theorem 1. Under the assumptions $(\mathrm{H} 2)$ and $(\mathbf{H 3})$, the mapping (13) considered in the weighted Hölder spaces

$$
S: \Lambda_{\beta}^{0, \alpha}(\Omega) \times C^{2, \alpha}(\partial \Omega) \longmapsto \Lambda_{\beta}^{2, \alpha}(\Omega)
$$

is an isomorphism if and only if $\beta-\alpha \in(2,3)$.

The following result on asymptotics is due to (Kondratiev, 1967; Mazja and Plamenevskii, 1978) (see also (Mazja and Plamenevskii, 1973) and, e.g., (Nazarov and Plamenevsky, 1994)).

Theorem 2. If the right hand side in (11) $\mathcal{F} \in \Lambda_{\gamma}^{0, \alpha}(\Omega)$ and $\gamma-\alpha \in(1,2)$, then the solution $V$ to (11) can be decomposed into $V(x)=\widetilde{V}(x)+V(\mathcal{O})$ and the following estimate holds:

$$
\begin{aligned}
|V(\mathcal{O})|+ & \left\|\tilde{V} ; \Lambda_{\gamma}^{2, \alpha}(\Omega)\right\| \\
& \leq c\left(\left\|\mathcal{F} ; \Lambda_{\gamma}^{0, \alpha}(\Omega)\right\|+\left\|g ; C^{2, \alpha}(\partial \Omega)\right\|\right) .
\end{aligned}
$$

An assertion, similar to Theorem 1, is valid for the perforated domain $\Omega(\varepsilon)$ as well. The following result is due to (Mazja et al., 1981) (see also (Mazja et al., 1991; Nazarov and Plamenevsky, 1994))

Theorem 3. Under the assumptions (H2) and (H3), the linearized problem

$$
\begin{cases}-\Delta_{x} v^{\varepsilon}(x)-F_{v}^{\prime}(x, v(x)) v^{\varepsilon}(x)=\mathcal{F}^{\varepsilon}(x), & x \in \Omega(\varepsilon), \\ v^{\varepsilon}(x)=g^{\varepsilon}(x), & x \in \partial \Omega(\varepsilon)\end{cases}
$$

is uniquely solvable and the solution operator

$$
S_{\varepsilon}:\left\{\mathcal{F}^{\varepsilon}, g^{\varepsilon}\right\} \longmapsto v^{\varepsilon}
$$

is bounded in the weighted Hölder spaces

$$
S_{\varepsilon}: \Lambda_{\beta}^{0, \alpha}(\Omega(\varepsilon)) \times \Lambda_{\beta}^{2, \alpha}(\partial \Omega(\varepsilon)) \longmapsto \Lambda_{\beta}^{2, \alpha}(\Omega(\varepsilon)) .
$$

Moreover, in the case when $\beta-\alpha \in(2,3)$ the estimate

$$
\begin{aligned}
& \left\|v^{\varepsilon} ; \Lambda_{\beta}^{2, \alpha}(\Omega(\varepsilon))\right\| \\
& \leq c_{\beta}\left(\left\|\mathcal{F}^{\varepsilon} ; \Lambda_{\beta}^{0, \alpha}(\Omega(\varepsilon))\right\|+\left\|g^{\varepsilon} ; \Lambda_{\beta}^{2, \alpha}(\partial \Omega(\varepsilon))\right\|\right)
\end{aligned}
$$

is valid, where the constant $c_{\beta}$ is independent of $\varepsilon \in$ $\left(0, \varepsilon_{0}\right]$.

Remark 1. Since $|x| \geq c \varepsilon>0$ in $\Omega(\varepsilon)$, the weighted norm $\left\|\cdot ; \Lambda_{\beta}^{2, \alpha}(\Omega(\varepsilon))\right\|$ is equivalent to the usual norm $\left\|\cdot ; C^{2, \alpha}(\Omega(\varepsilon))\right\|$. However; the equivalence constants depend on $\varepsilon$. Thus $\Lambda_{\beta}^{2, \alpha}(\Omega(\varepsilon))$ and $C^{2, \alpha}(\Omega(\varepsilon))$ coincide algebraically and topologically but are normed in a different way. The norm of the operator $S_{\varepsilon}$ is uniformly bounded for $\varepsilon \in\left(0, \varepsilon_{0}\right]$ for any $\beta$, although the constant $c_{\beta}$ in (17) depends on $\varepsilon$ provided $\beta \notin(2,3)$, that is, the norm of the inverse operator is uniformly bounded in $\varepsilon \in\left(0, \varepsilon_{1}\right]$ only in the case of $\beta \in(2,3)$.

For the nonlinear problem (3), we shall use the classical solutions to the boundary value problem (3), which means that for given $F \in C^{0, \alpha}(\bar{\Omega} \times \mathbb{R}), \alpha \in(0,1)$, the solution lives in $C^{2, \alpha}(\bar{\Omega})$. We refer to (Ladyzhenskaya and Ural'tseva, 1968; Gilbarg and Trudinger, 2001) for a result on the existence and uniqueness of solutions to semilinear elliptic boundary value-problems. This means, in particular, that the problem (3) admits a unique solution $u^{\varepsilon} \in C^{2, \alpha}(\Omega(\varepsilon))$ for some $0<\alpha<1$ and for all $\varepsilon \in\left[0, \varepsilon_{0}\right)$.

\section{Topological derivative for semilinear problems in 3D}

We present here a complete analysis of the semilinear elliptic problem in three spatial dimensions. Such an analysis is interesting on its own, since in the existing literature there is no elementary derivation of the form of topological derivatives for nonlinear problems besides (Mazja et al., 1981), (see also (Mazja et al., 1991)), i.e., using asymptotic approximations of solutions to nonlinear PDEs. There are some results on topological derivatives of the shape functional for nonlinear problems, see, e.g., (Amstutz, 2006). However, such results are given in terms of one term exterior approximation of the solutions and without an asymtotically sharp estimate.

2.1. Formal asymptotic analysis. Referring to (Mazja et al., 1991), we set

$$
u^{\varepsilon}(x)=v(x)+w\left(\varepsilon^{-1} x\right)+\varepsilon v^{\prime}(x)+\cdots,
$$

where $v, v^{\prime}$ and $w$ are components of regular and boundary layer types, respectively. Thus,

$$
\begin{aligned}
-\Delta_{x} v & (x)-\varepsilon^{-2} \Delta_{\xi} w(\xi)-\varepsilon \Delta_{x} v^{\prime}(x)+\cdots \\
= & F\left(x, v(x)+w\left(\varepsilon^{-1} x\right)+\varepsilon v^{\prime}(x)+\cdots\right) \\
= & F(x, v(x))+\left(w\left(\varepsilon^{-1} x\right)\right. \\
& \left.+\varepsilon v^{\prime}(x)\right) F_{v}^{\prime}(x, v(x))+\cdots .
\end{aligned}
$$

In view of (7), the first terms on the left and right-hand sides are cancelled and, moreover, $w$ satisfies the problem (6) with $u(\mathcal{O})=v(\mathcal{O})$,

$$
\left\{\begin{aligned}
-\Delta_{\xi} w(\xi) & =0, & & \xi \in \mathbb{R}^{3} \backslash \bar{\omega} \\
w(\xi) & =-v(\mathcal{O}), & & \xi \in \partial \omega,
\end{aligned}\right.
$$

while the boundary datum comes from the relation

$$
\begin{aligned}
v(x)+w & \left(\varepsilon^{-1} x\right)+\varepsilon v^{\prime}(x) \\
& =v(\mathcal{O})+w\left(\varepsilon^{-1} x\right)+O(\varepsilon), \quad x \in \partial \omega_{\varepsilon} .
\end{aligned}
$$


We have

$$
w(\xi)=-v(\mathcal{O}) P(\xi),
$$

where $P$ is the capacity potential (Landkof, 1966; Pólya and Szegö, 1951), e.g., a harmonic function in $\mathbb{R}^{3} \backslash \bar{\omega}$ such that $P(\xi)=1$ on $\partial \omega$ and

$$
P(\xi)=|\xi|^{-1} \operatorname{cap}(\omega)+O\left(|\xi|^{-2}\right),
$$

where $\operatorname{cap}(\omega)$ is the capacity of the set $\bar{\omega}$. Since

$$
w\left(\varepsilon^{-1} x\right)=-|x|^{-1} \varepsilon v(\mathcal{O}) \operatorname{cap}(\omega)+O\left(\varepsilon^{2}|x|^{-2}\right),
$$

we collect coefficients on $\varepsilon$ in (19) and obtain

$$
\left\{\begin{aligned}
-\Delta_{x} v^{\prime}(x)-v^{\prime}(x) F_{v}^{\prime}(x, v(x)) & \\
=-a \Phi(x) F_{v}^{\prime}(x, v(x)), & x \in \Omega, \\
v^{\prime}(x)=a \Phi(x), & x \in \partial \Omega,
\end{aligned}\right.
$$

where $a=4 \pi v(\mathcal{O}) \operatorname{cap}(w)$ and $\Phi(x)=(4 \pi|x|)^{-1}$ is the fundamental solution of the Laplace equation in $\mathbb{R}^{3}$.

Since a direct calculation yields $F^{\prime}(\cdot, v) \Phi \in$ $\Lambda_{\gamma}^{0, \alpha}(\Omega)$ with any $\gamma>1+\alpha$, we obtain the solution $v^{\prime} \in \Lambda_{\beta}^{2, \alpha}(\Omega)$ of the problem (24) such that $v^{\prime}-v^{\prime}(\mathcal{O}) \in$ $\Lambda_{\gamma}^{2, \alpha}(\Omega)$ where $\beta-\alpha \in(2,3)$ and $\gamma-\alpha \in(1,2)$ can be taken arbitrarily in the prescribed intervals.

2.2. Justification of asymptotic. We search for a solution of the problem (3) in the form

$$
u^{\varepsilon}(x)=v(x)+w\left(\varepsilon^{-1} x\right)+\varepsilon v^{\prime}(x)+\hat{u}^{\varepsilon}(x),
$$

where $\hat{u}^{\varepsilon}$ is a small remainder, satisfying the problem

$$
\left\{\begin{aligned}
-\Delta_{x} \hat{u}^{\varepsilon}(x) & =\hat{\mathcal{F}}^{\varepsilon}(x ; \hat{u}), & & x \in \Omega(\varepsilon), \\
\hat{u}^{\varepsilon}(x) & =\hat{g}_{\Omega}^{\varepsilon}(x), & & x \in \partial \Omega, \\
\hat{u}^{\varepsilon}(x) & =\hat{g}_{\omega}^{\varepsilon}(x), & & x \in \partial \omega(\varepsilon) .
\end{aligned}\right.
$$

Here

$$
\begin{aligned}
\hat{\mathcal{F}}^{\varepsilon}(x ; \hat{u})= & F\left(x, v(x)+w\left(\varepsilon^{-1} x\right)+\varepsilon v^{\prime}(x)\right. \\
& \left.+\hat{u}^{\varepsilon}(x)\right)-F(x, v(x)) \\
& -\varepsilon\left(v^{\prime}(x)-a \Phi(x)\right) F_{v}^{\prime}(x, v(x)), \\
\hat{g}_{\Omega}^{\varepsilon}(x)= & -w\left(\varepsilon^{-1} x\right)-a \varepsilon \Phi(x), \\
\hat{g}_{\omega}^{\varepsilon}(x)= & -v(x)+v(\mathcal{O})-\varepsilon v^{\prime}(x) .
\end{aligned}
$$

We are going to employ the Banach contraction principle and, thus, we need to estimate the norms of (27).

Owing to 21), 22), the function $x \mapsto w\left(\varepsilon^{-1} x\right)+$ $a \varepsilon \Phi(x)$ is smooth on the surface $\partial \Omega$, where $|x| \geq c>0$, and

$$
\begin{aligned}
\mid w\left(\varepsilon^{-1} x\right) & +a \varepsilon \Phi(x) \mid \\
& \leq\left.|v(\mathcal{O})||P(\xi)-\operatorname{cap}(\omega)| \xi\right|^{-1} \mid \\
& \leq c \varepsilon^{2}|x|^{-2} \leq c \varepsilon^{2}, \\
\mid \nabla_{x}^{k} w\left(\varepsilon^{-1} x\right) & +a \varepsilon \nabla_{x}^{k} \Phi(x) \mid \\
& \leq\left.\varepsilon^{-k}|v(\mathcal{O})|\left|\nabla_{\xi}^{k} P(\xi)-\operatorname{cap}(\omega) \nabla_{\xi}^{k}\right| \xi\right|^{-1} \mid \\
& \leq c \varepsilon^{-k}|\xi|^{-2-k}=c \varepsilon^{2}|x|^{-2-k} \leq c \varepsilon^{2} .
\end{aligned}
$$

Hence, by the above inequalities for the function $x \mapsto w\left(\varepsilon^{-1} x\right)+a \varepsilon \Phi(x)$, we obtain the following estimates of the norm of $\hat{g}_{\Omega}^{\varepsilon}$ in the weighted Hölder space :

$$
\begin{aligned}
\left\|\hat{g}_{\Omega}^{\varepsilon} ; \Lambda_{\beta}^{2, \alpha}(\partial \Omega)\right\| & \leq c\left\|\hat{g}_{\Omega}^{\varepsilon} ; C^{2, \alpha}(\partial \Omega)\right\| \\
& \leq c\left\|\hat{g}_{\Omega}^{\varepsilon} ; C^{3}(\partial \Omega)\right\| \leq c \varepsilon^{2} .
\end{aligned}
$$

Moreover, for $\beta-\beta^{\prime}>0$, we have

$$
\begin{aligned}
\| \hat{g}_{\omega}^{\varepsilon} ; & \Lambda_{\beta}^{2, \alpha}(\partial \Omega) \| \\
\leq & c\left(\operatorname { s u p } _ { x \in \partial \omega _ { \varepsilon } } \sum _ { k = 0 } ^ { 2 } | x | ^ { \beta - 2 - \alpha + k } \left(\left|\nabla_{x}^{k}(v(x)-v(\mathcal{O}))\right|\right.\right. \\
& \left.+\varepsilon\left|\nabla_{x}^{k} v^{\prime}(x)\right|\right)+\sup _{x, y \in \partial \omega_{\varepsilon}}|x|^{\beta}|x-y|^{-\alpha}\left(\mid \nabla_{x}^{2} v(x)\right. \\
& \left.\left.\quad-\nabla_{y}^{2} v(y)|+\varepsilon| \nabla_{x}^{2} v^{\prime}(x)-\nabla_{y}^{2} v^{\prime}(y) \mid\right)\right) \\
\leq & c\left(\varepsilon^{\beta-1-\alpha}\left\|v ; C^{2, \alpha}(\Omega)\right\|+\varepsilon^{1+\beta-\beta^{\prime}}\left\|v^{\prime} ; \Lambda_{\beta^{\prime}}^{2, \alpha}(\Omega)\right\|\right) .
\end{aligned}
$$

Notice that $v^{\prime} \in \Lambda_{\beta^{\prime}}^{2, \alpha}(\partial \Omega)$ with arbitrary $\beta^{\prime} \in(2+$ $\alpha, 3+\alpha)$. We shall further select the indices $\beta$ and $\beta^{\prime}$ in an appropriate way.

Write

$$
\begin{aligned}
\mathbf{F}(x, V(x))= & F(x, v(x)+V(x)) \\
& -F(x, v(x))-V(x) F_{v}^{\prime}(x, v(x)),
\end{aligned}
$$

so that

$$
\begin{aligned}
& \hat{\mathcal{F}}^{\varepsilon}\left(x ; \hat{u}^{\varepsilon}\right) \\
& =\mathbf{F}\left(x, w\left(\varepsilon^{-1} x\right)+\varepsilon v^{\prime}(x)+\hat{u}^{\varepsilon}(x)\right) \\
& \quad+\left(w\left(\varepsilon^{-1} x\right)+\varepsilon a \Phi(x)+\hat{u}^{\varepsilon}(x)\right) F_{v}^{\prime}(x, v(x)) .
\end{aligned}
$$

Since $\left(x \mapsto F_{v}^{\prime}(x, v(x))\right) \in C^{0, \alpha}(\Omega)$, by (H3), we take into account the representation (22) together with the inequality $\beta-\alpha>2$ and, as a result, we obtain

$$
\begin{aligned}
&\left\|(w+\varepsilon a \Phi) F_{v}^{\prime} ; \Lambda_{\beta}^{0, \alpha}(\Omega(\varepsilon))\right\| \\
& \leq c\left(\sup _{x \in \Omega(\varepsilon)}|x|^{\beta-\alpha}\left(\frac{|x|}{\varepsilon}\right)^{-2}\right. \\
&+\left(\sup _{x, y \in \Omega(\varepsilon),|x-y|<|x| / 2}|x|^{\beta}|x-y|^{-\alpha}\right. \\
& \\
&\left.\cdot\left|\frac{x}{\varepsilon}-\frac{y}{\varepsilon}\right|\left(\frac{|x|}{\varepsilon}\right)^{-3}\right) \\
& \leq c \varepsilon^{2} \sup _{x \in \Omega(\varepsilon)}\left(|x|^{\beta-\alpha}|x|^{-2}+|x|^{\beta+1-\alpha}|x|^{-3}\right) \\
& \leq c \varepsilon^{2} .
\end{aligned}
$$

To estimate the first term on the right-hand side of (32), we need the following assumption on $\mathbf{F}$ : 
(H4) With a certain $\kappa \in(0,1)$ and for $|V(x)| \leq C$, $x \in \Omega$, the inequality $|\mathbf{F}(x, V(x))| \leq c|V(x)|^{1+\kappa}$ and the following relations are valid:

$$
\begin{aligned}
& \left|\mathbf{F}\left(x, V_{1}(x)\right)-\mathbf{F}\left(y, V_{2}(y)\right)\right| \\
& \quad \leq c\left(|x-y|^{\alpha}\left(\left|V_{1}(x)\right|+\left|V_{2}(y)\right|\right)^{1+\kappa}\right. \\
& \left.\quad+\left|V_{1}(x)-V_{2}(y)\right|\left(\left|V_{1}(x)\right|^{\kappa}+\left|V_{2}(y)\right|^{\kappa}\right)\right), \\
& \mid \mathbf{F}\left(x, V_{1}(x)\right)-\mathbf{F}\left(x, V_{2}(x)\right) \\
& \quad-\left(\mathbf{F}\left(y, V_{1}(y)\right)-\mathbf{F}\left(y, V_{2}(y)\right)\right) \mid \\
& \leq c\left(\left|V_{1}(x)-V_{2}(x)-\left(V_{1}(y)-V_{2}(y)\right)\right| \mathbf{V}(x, y)^{\kappa}\right. \\
& \quad+|x-y|^{\alpha}\left(\left|V_{1}(x)-V_{2}(x)\right|\right. \\
& \left.\quad+\left|V_{1}(y)-V_{2}(y)\right|\right) \mathbf{V}(x, y)^{\kappa} \\
& +\left(\left|V_{1}(x)-V_{2}(x)\right|\right. \\
& \left.+\left|V_{1}(y)-V_{2}(y)\right|\right)\left(\left|V_{1}(x)-V_{2}(y)\right|\right. \\
& \left.+\left|V_{1}(y)-V_{2}(y)\right|\right)(1+\mathbf{V}(x, y))^{\kappa-1},
\end{aligned}
$$

where

$$
\mathbf{V}(x, y)=\left|V_{1}(x)\right|+\left|V_{2}(x)\right|+\left|V_{1}(y)\right|+\left|V_{2}(y)\right| .
$$

In other words, the mapping $\mathbf{F}$ satisfies the Hölder condition in both arguments and has a power-law growth in the second one. Moreover, the second order difference satisfies the estimate (34).

Lemma 1. (1) Let $V \in \Lambda_{\beta}^{2, \alpha}(\Omega(\varepsilon))$ and $\beta-\alpha \in$ $(2,3), \alpha \in(0,1), \kappa \in(0,1)$. Then, for $x \in \Omega(\varepsilon)$ and $|x-y|<|x| / 2$, the estimates

$$
\begin{array}{r}
|x|^{\beta-\alpha}|V(x)|^{1+\kappa} \leq c\left\|V ; \Lambda_{\beta}^{2, \alpha}(\Omega(\varepsilon))\right\|^{1+\kappa} \\
\left.|x|^{\beta-(\beta-\alpha) \frac{\kappa}{1+\kappa} \mid x-} y\right|^{-\alpha}|V(x)-V(y)|^{1+\kappa} \\
\leq c\left\|V ; \Lambda_{\beta}^{2, \alpha}(\Omega(\varepsilon))\right\|^{1+\kappa}
\end{array}
$$

are valid.

(2) Under the same restrictions on $\alpha, \beta, \kappa$ and $x, y$ as above,

$$
\begin{aligned}
& |x|^{\beta-\alpha}\left|w\left(\varepsilon^{-1} x\right)\right|^{1+\kappa} \leq c \varepsilon^{1+\kappa}, \\
& |x|^{\beta-(\beta-\alpha) \frac{\kappa}{1+\kappa}}|x-y|^{-\alpha}\left|w\left(\varepsilon^{-1} x\right)-w\left(\varepsilon^{-1} y\right)\right|^{1+\kappa} \\
& \leq c \varepsilon \text {. }
\end{aligned}
$$

Proof. First, we readily show the first assertion:

$$
\begin{aligned}
& |x|^{\beta-\alpha}|V(x)|^{1+\kappa} \\
& \quad \leq|x|^{\beta-\alpha}|x|^{-(1+\kappa)(\beta-2-\alpha)}\left(|x|^{\beta-2-\alpha}|V(x)|\right)^{1+\kappa} \\
& \quad \leq|x|^{2-\kappa(\beta-2-\alpha)}\left\|V ; \Lambda_{\beta}^{2, \alpha}(\Omega(\varepsilon))\right\|^{1+\kappa}
\end{aligned}
$$

The second inequality follows from the relation

$$
2-\kappa(\beta-2-\alpha) \geq 2-1(3-2-\alpha)>1>0 .
$$

Since

$$
\frac{1}{2}|x|<|y|<\frac{3}{2}|x|
$$

in view of

$$
|x-y|<\frac{x}{2}
$$

and using the Newton-Leibnitz formula, we conclude that

$$
\begin{aligned}
& |x|^{\beta-(\beta-\alpha) \frac{\kappa}{1+\kappa}}|x-y|^{-\alpha}|V(x)-V(y)|^{1+\kappa} \\
& \leq c|x|^{\beta-(\beta-\alpha) \frac{\kappa}{1+\kappa}}|x-y|^{-\alpha}|x|^{-\beta+1+\alpha}|x-y| \\
& \text {. } \sup _{x \in \Omega(\varepsilon)}\left(|x|^{\beta-1-\alpha}\left|\nabla_{x} V(x)\right|\right) \\
& \leq c|x|^{\beta-(\beta-\alpha) \frac{\kappa}{1+\kappa}}|x|^{1-\alpha}|x|^{-\beta+1+\alpha}\left\|V ; \Lambda_{\beta}^{2, \alpha}(\Omega(\varepsilon))\right\|
\end{aligned}
$$

while applying the inequalities

$$
\begin{aligned}
& \beta-(\beta-\alpha) \frac{\kappa}{1+\kappa}+1-\alpha-\beta+1+\alpha \\
& \quad=2-(\beta-\alpha) \frac{\kappa}{1+\kappa} \geq \frac{2-(\beta-\alpha-2) \kappa}{1+\kappa}>0 .
\end{aligned}
$$

Based on the assumptions $\beta-\alpha>2$ and $1+\kappa<2$, we prove the second assertion. We have

$$
\begin{aligned}
|x|^{\beta-\alpha}\left|w\left(\varepsilon^{-1} x\right)\right|^{1+\kappa} & \leq c|x|^{\beta-\alpha}\left(1+\frac{|x|}{\varepsilon}\right)^{-1-\kappa} \\
& =c \varepsilon^{1+\kappa} \frac{|x|^{\beta-\alpha}}{(\varepsilon+|x|)^{1+\kappa}} \leq c \varepsilon^{1+\kappa} .
\end{aligned}
$$

Owing to the estimate $|P(\xi)| \leq c(1+|\xi|)^{-1}$ for the capacity potential and the boundary condition (21), it follows that

$$
\begin{aligned}
& |x|^{\beta-(\beta-\alpha) \frac{\kappa}{1+\kappa}}|x-y|^{-\alpha}\left|w\left(\varepsilon^{-1} x\right)-w\left(\varepsilon^{-1} y\right)\right| \\
& \leq c|x|^{\beta-(\beta-\alpha) \frac{\kappa}{1+\kappa}}|x-y|^{-\alpha}\left|\frac{x}{\varepsilon}-\frac{y}{\varepsilon}\right|\left(1+\frac{|x|}{\varepsilon}\right)^{-2} \\
& \left.\cdot \sup _{\xi \in \mathbb{R}^{3} \backslash \omega}(1+|\xi|)^{2}\left|\nabla_{\xi} w(\xi)\right|\right) \\
& \leq c \varepsilon|x|^{\beta-(\beta-\alpha) \frac{\kappa}{1+\kappa}}|x|^{1-\alpha}(\varepsilon+|x|)^{-2} \\
& \leq c \varepsilon \text {. }
\end{aligned}
$$

Indeed, in the first inequality we have again applied the Newton-Leibnitz formula, and in the second one we have used the fact that $\left|\nabla_{\xi} P(\xi)\right| \leq c(1+|\xi|)^{-2}$ and

$$
\beta-\alpha-(\beta-\alpha) \frac{\kappa}{1+\kappa}=\frac{\beta-\alpha}{1+\kappa} \geq 1 \text {. }
$$

We now list the necessary estimates based on Lemma 1 and (H5). We start with the boundedness of the first term in 32 multiplied by a weight. We obtain

$$
\begin{aligned}
& |x|^{\beta-\alpha}\left|\mathbf{F}\left(x, w\left(\varepsilon^{-1} x\right)+\varepsilon v^{\prime}(x)+\hat{u}^{\varepsilon}(x)\right)\right| \\
& \leq c|x|^{\beta-\alpha}\left(\left|w\left(\varepsilon^{-1} x\right)\right|^{1+\kappa}+\varepsilon^{1+\kappa}\left|v^{\prime}(x)\right|^{1+\kappa}\right. \\
& \left.\left.\quad+\mid \hat{u}^{\varepsilon}(x)\right)\left.\right|^{1+\kappa}\right) \\
& \leq c\left(\varepsilon^{1+\kappa}+\left\|\hat{u}^{\varepsilon} ; \Lambda_{\beta}^{2, \alpha}(\Omega(\varepsilon))\right\|^{1+\kappa}\right) .
\end{aligned}
$$


Second, we verify the boundedness of the weighted difference, namely,

$$
\begin{aligned}
|x|^{\beta}|x-y|^{-\alpha} \mid \mathbf{F}(x, \overbrace{w\left(\varepsilon^{-1} x\right)+\varepsilon v^{\prime}(x)+\hat{u}^{\varepsilon}(x)}^{=V(x)}) \\
\quad-\left.\mathbf{F}(y, V(y))|\leq c| x\right|^{\beta}\left(|V(x)|^{1+\kappa}+|x-y|^{-\alpha} \mid V(x)\right. \\
\left.\quad-V(y) \mid\left(|V(x)|^{\kappa}+|V(y)|^{\kappa}\right)\right) \\
\leq c\left(\varepsilon^{1+\kappa}+\left\|\hat{u}^{\varepsilon} ; \Lambda_{\beta}^{2, \alpha}(\Omega(\varepsilon))\right\|^{1+\kappa}\right. \\
\quad+\left(\varepsilon^{\kappa}+\left\|\hat{u}^{\varepsilon} ; \Lambda_{\beta}^{2, \alpha}(\Omega(\varepsilon))\right\|^{\kappa}\right)|x|^{\beta-(\beta-\alpha) \frac{\kappa}{1+\kappa}}|x-y|^{-\alpha} \\
\quad \cdot\left\{\left|w\left(\frac{x}{\varepsilon}\right)-w\left(\frac{y}{\varepsilon}\right)\right|\right. \\
\left.\left.\quad+\varepsilon\left|v^{\prime}(x)-v^{\prime}(y)\right|+\left|\hat{u}^{\varepsilon}(x)-\hat{u}^{\varepsilon}(y)\right|\right\}\right) \\
\leq c\left(\varepsilon^{1+\kappa}+\left\|\hat{u}^{\varepsilon} ; \Lambda_{\beta}^{2, \alpha}(\Omega(\varepsilon))\right\|^{1+\kappa}\right) .
\end{aligned}
$$

Now, we deduce the local Lipschitz continuity of the first part of the mapping (32):

$$
\begin{aligned}
& |x|^{\beta-\alpha} \mid \mathbf{F}(x, \overbrace{w\left(\varepsilon^{-1} x\right)+\varepsilon v^{\prime}(x)+\hat{u}_{1}^{\varepsilon}(x)}^{=V_{1}(x)}) \\
& -\mathbf{F}(x, \overbrace{w\left(\varepsilon^{-1} x\right)+\varepsilon v^{\prime}(x)+\hat{u}_{2}^{\varepsilon}(x)}) \mid \\
& \leq c|x|^{\beta-\alpha}\left|\hat{u}_{1}^{\varepsilon}(x)-\hat{u}_{2}^{\varepsilon}(x)\right|\left(\left|V_{1}(x)\right|^{\kappa}+\left|V_{2}(x)\right|^{\kappa}\right) \\
& \leq c\left\|\hat{u}_{1}^{\varepsilon}-\hat{u}_{2}^{\varepsilon} ; \Lambda_{\beta}^{2, \alpha}(\Omega(\varepsilon))\right\|\left(\varepsilon^{\kappa}+\left\|\hat{u}_{1}^{\varepsilon} ; \Lambda_{\beta}^{2, \alpha}(\Omega(\varepsilon))\right\|^{\kappa}\right. \\
& \left.\quad+\left\|\hat{u}_{2}^{\varepsilon} ; \Lambda_{\beta}^{2, \alpha}(\Omega(\varepsilon))\right\|^{\kappa}\right) .
\end{aligned}
$$

Finally, we prove the local Lipschitz continuity for the weighted second order differences of the mapping $\mathbf{F}$. For example, the first term on the right-hand side of (34) gets the bound

$$
\begin{aligned}
c|x|^{\beta-(\beta-\alpha) \frac{\kappa}{1+\kappa}}|x-y|^{-\alpha} \mid\left(V_{1}(x)-V_{2}(x)\right) \\
-\left(V_{1}(y)-V_{2}(y)\right) \mid\left(\varepsilon^{\kappa}+\left\|\hat{u}_{1}^{\varepsilon} ; \Lambda_{\beta}^{2, \alpha}(\Omega(\varepsilon))\right\|\right. \\
\left.+\left\|\hat{u}_{2}^{\varepsilon} ; \Lambda_{\beta}^{2, \alpha}(\Omega(\varepsilon))\right\|\right) \\
\quad \leq c \| \hat{u}_{1}^{\varepsilon}-\hat{u}_{2}^{\varepsilon} ; \Lambda_{\beta}^{2, \alpha} \\
\quad \cdot(\Omega(\varepsilon)) \|\left(\varepsilon^{\kappa}+\left\|\hat{u}_{1}^{\varepsilon} ; \Lambda_{\beta}^{2, \alpha}(\Omega(\varepsilon))\right\|^{\kappa}\right) .
\end{aligned}
$$

The other two terms in (34) are estimated in the same way as in (35) and (36), respectively.

The above estimates allow us to apply the Banach fixed point theorem to verify the existence of the remainder $\hat{u}^{\varepsilon}$. To this end, we rewrite problem (26) in the form of an abstract equation in the Banach space $\mathfrak{R}=$ $\Lambda_{\beta}^{2, \alpha}(\Omega(\varepsilon))$, namely,

$$
\hat{u}^{\varepsilon}=\mathfrak{G} \hat{u}^{\varepsilon}
$$

where

$$
\mathfrak{G} \hat{u}^{\varepsilon}=\mathcal{S}_{\epsilon}\left(\hat{\mathcal{F}}^{\varepsilon}\left(. ; \hat{u}^{\varepsilon}\right), \hat{g}_{\Omega}^{\varepsilon}, \hat{g}_{\omega}^{\varepsilon}\right)
$$

and $S_{\varepsilon}$ denotes the isomorphism (16). Let $\hat{u}^{\varepsilon}$ belong to the ball $\mathcal{B} \subset \mathfrak{R}$ of radius $\mathfrak{C} \varepsilon^{1+\kappa}$. We further need to verify two properties. First, the mapping $\mathfrak{C}$ maps the ball $\mathcal{B}$ into itself,

$$
\mathcal{B} \ni \hat{u}^{\varepsilon} \Rightarrow \mathfrak{G} \hat{u}^{\varepsilon} \in \mathcal{B},
$$

and second, the mapping becomes a strict contraction on the ball, i.e.,

$\|\mathfrak{G} \mathfrak{v}-\mathfrak{G} \mathfrak{w} ; \mathfrak{R}\| \leq k\|\mathfrak{v}-\mathfrak{w} ; \mathfrak{R}\|, \quad \mathfrak{v}, \mathfrak{w} \in \mathfrak{R}$ with $k<1$

By (29), 30), (33) and (35), (36), we have

$$
\begin{aligned}
\left\|\mathfrak{G} \hat{u}^{\varepsilon} ; \Re\right\| \leq & c\left(\left\|\hat{\mathcal{F}}^{\varepsilon} ; \Lambda_{\beta}^{0, \alpha}(\Omega(\varepsilon))\right\|+\left\|\hat{g}_{\Omega}^{\varepsilon} ; \Lambda_{\beta}^{2, \alpha}(\Omega(\varepsilon))\right\|\right. \\
& \left.+\left\|\hat{g}_{\omega}^{\varepsilon} ; \Lambda_{\beta}^{2, \alpha}(\Omega(\varepsilon))\right\|\right) \\
\leq & c\left(\varepsilon^{1+\kappa}+\left\|\hat{u}^{\varepsilon} ; \Re\right\|^{1+\kappa}\right. \\
& \left.+\varepsilon^{2}+\varepsilon^{\beta-1-\alpha}+\varepsilon^{1+\beta-\beta^{\prime}}\right) .
\end{aligned}
$$

Let us fix $\beta, \alpha$ and $\beta^{\prime}, \kappa$ such that

$$
\begin{gathered}
(1,2) \ni \beta-\alpha-1 \geq 1+\kappa, \\
\beta-\beta^{\prime} \geq \kappa .
\end{gathered}
$$

Recall that $\beta-\alpha$ and $\beta^{\prime}-\alpha$ belong to the interval $(2,3)$. Thus, to satisfy (44), we must put $\beta-\alpha$ near 3 (satisfying (43) as well) and $\beta^{\prime}-\alpha$ near 2 . This allows us to create a gap of any length $\kappa \in(0,1)$.

If (43) and 44) hold true, we obtain

$$
\left\|\mathfrak{G} \hat{u}^{\varepsilon} ; \mathfrak{R}\right\| \leq c\left(4 \varepsilon^{1+\kappa}+\left\|\hat{u}^{\varepsilon} ; \mathfrak{R}\right\|^{1+\kappa}\right) \leq \mathfrak{C} \varepsilon^{1+\kappa},
$$

while the desired inequality $\mathfrak{C} \geq c\left(4+\mathfrak{C}^{1+\kappa} \varepsilon^{(1+\kappa) \kappa}\right)$ is achieved by a proper choice of the constant $\mathfrak{C}$ (e.g., $\mathfrak{C}=$ $5 c$ ) and the bound for the parameter $\varepsilon_{0}$ in the condition $\varepsilon \in\left(0, \varepsilon_{0}\right]$.

By virtue of (37) and 38, the estimate

$$
\|\mathfrak{G} \mathfrak{v}-\mathfrak{G} \mathfrak{w} ; \mathfrak{R}\| \leq \underbrace{c\left(\varepsilon^{\kappa}+2 \mathfrak{C}^{\kappa} \varepsilon^{(1+\kappa) \kappa}\right)}_{k}\|v-w ; \mathfrak{R}\|
$$

is valid. The necessary relation $k<1$ can be achieved by diminishing, if necessary, the upper bound $\varepsilon_{0}$ for $\varepsilon$ again.

Theorem 4. Let the indices $\beta, \alpha$ and $\kappa \in(0,1)$ satisfy (43) and $\beta-2>\kappa$, while (H2) and (H4) hold true. Then there exist positive constants $\mathfrak{C}$ and $\varepsilon_{0}$ such that, for $\varepsilon \in$ $\left(0, \varepsilon_{0}\right]$, the non-linear problem (26) has a unique small solution $\hat{u}^{\varepsilon}$, namely,

$$
\left\|\hat{u}^{\varepsilon} ; \Lambda_{\beta}^{2, \alpha}(\Omega(\varepsilon))\right\| \leq \mathfrak{C} \varepsilon^{1+\kappa} .
$$

Consequently, the singularity perturbed problem (3) has at least one solution (25). 
In the theorem we have proven the existence of a small remainder $\hat{u}^{\varepsilon}$ in (25), i.e., we have verified that the problem (3) has a unique solution in a small ball centred at the approximate asymptotic solution. If the uniqueness of the solution $\hat{u}^{\varepsilon}$ is known, for example, $F$ in (3) gives rise to a monotone operator, the remainder is unique without any smallness assumption.

2.3. Formal asymptotic of the shape functional. We have

$$
\begin{aligned}
\mathcal{J}\left(u^{\varepsilon} ; \Omega(\varepsilon)\right) & \\
= & \int_{\Omega(\varepsilon)} J(x, v(x)) \mathrm{d} x \\
& +\int_{\Omega(\varepsilon)}\left(w\left(\varepsilon^{-1} x\right)+\varepsilon v^{\prime}(x)\right) J_{v}^{\prime}(x, v(x)) \mathrm{d} x+\cdots \\
= & \int_{\Omega} J(x, v(x)) \mathrm{d} x \\
& +\varepsilon \int_{\Omega}\left(v^{\prime}(x)-a \Phi(x)\right) J_{v}^{\prime}(x, v(x)) \mathrm{d} x+\cdots
\end{aligned}
$$

We now introduce the following assumption:

(H5) $J \in C^{0, \alpha}(\Omega \times \mathbb{R}), J_{v}^{\prime} \in C^{0, \alpha}(\Omega \times \mathbb{R})$.

Let $p \in C^{2, \alpha}(\Omega)$ be a solution of the problem

$$
\left\{\begin{array}{rlrl}
-\Delta_{x} p(x)-F_{v}^{\prime}(x, v(x)) p(x) & & \\
& =J_{v}^{\prime}(x, v(x)), & & x \in \Omega, \\
p(x) & =0, & & x \in \partial \Omega .
\end{array}\right.
$$

Integrating by parts in $\Omega \backslash \mathbb{B}_{\delta}=\{x \in \Omega:|x|>\delta\}$ yields

$$
\begin{aligned}
\int_{\Omega} & \left(v^{\prime}(x)-a \Phi(x)\right) J_{v}^{\prime}(x, v(x)) \mathrm{d} x \\
= & -\lim _{\delta \rightarrow 0} \int_{\Omega \backslash \mathbb{B}_{\delta}}\left(\Delta_{x} p(x)+F_{v}^{\prime}(x, v(x)) p(x)\right) \\
& \cdot\left(v^{\prime}(x)-a \Phi(x)\right) \mathrm{d} x \\
= & -\lim _{\delta \rightarrow 0} \int_{\Omega \backslash \mathbb{B}_{\delta}} p(x)\left(\Delta_{x}+F_{v}^{\prime}(x, v(x))\right)\left(v^{\prime}(x)\right. \\
& -a \Phi(x)) \mathrm{d} x-\lim _{\delta \rightarrow 0} \int_{\partial \Omega} \partial_{n} p(x)\left(v^{\prime}(x)-a \Phi(x)\right) \mathrm{d} x \\
& +\lim _{\delta \rightarrow 0} \int_{\partial \mathbb{B}_{\delta}}\left(\partial_{|x|} p(x)\left(v^{\prime}(x)-a \Phi(x)\right)-p(x) \partial_{|x|}\left(v^{\prime}(x)\right.\right. \\
& -a \Phi(x))) \mathrm{d} x .
\end{aligned}
$$

By (24), we have $v^{\prime}(x)-a \Phi(x)=0$ for $x \in \Omega$ and

$$
\begin{aligned}
\left(\Delta_{x}+F_{v}^{\prime}(x,\right. & v(x)))\left(v^{\prime}(x)-a \Phi(x)\right) \\
= & \Delta_{x} v^{\prime}(x) \\
& +v^{\prime}(x) F_{v}^{\prime}(x, v(x))-a \Phi(x) F_{v}^{\prime}(x, v(x)) \\
= & 0 .
\end{aligned}
$$

On the other hand, $\partial_{|x|} p(x)\left(v^{\prime}(x)-a \Phi(x)\right)=O\left(\delta^{-1}\right)$ and, hence,

$$
\begin{aligned}
\int_{\Omega}\left(v^{\prime}(x)\right. & -a \Phi(x)) J_{v}^{\prime}(x, v(x)) \mathrm{d} x \\
- & \lim _{\delta \rightarrow 0} \int_{\partial \mathbb{B}_{\delta}}\left(\partial_{|x|} p(x)\left(v^{\prime}(x)-a \Phi(x)\right)\right. \\
- & \left.p(x) \partial_{|x|}\left(v^{\prime}(x)-a \Phi(x)\right)\right) \mathrm{d} x \\
& =-a \lim _{\delta \rightarrow 0} \int_{\partial \mathbb{B}_{\delta}} p(0)\left(4 \pi|x|^{2}\right)^{-1} \mathrm{~d} s_{x} \\
& =-a p(0)=-4 \pi v(\mathcal{O}) p(0) \operatorname{cap}(\omega) .
\end{aligned}
$$

Thus,

$$
\mathcal{J}\left(u^{\varepsilon} ; \Omega(\varepsilon)\right)=\mathcal{J}(v ; \Omega)-\varepsilon 4 \pi v(\mathcal{O}) p(0) \operatorname{cap}(\omega)+\cdots .
$$

Similarly to the first inequality in (H4), let the following assumption be valid:

(H6) With $\sigma \in(0,1)$,

$$
\begin{array}{r}
\left|J(x, v(x)+V(x))-J(x, v(x))-V(x) J_{v}^{\prime}(x, v(x))\right| \\
\leq c|V(x)|^{1+\sigma} .
\end{array}
$$

Using this assumption leads to the relation

$$
\begin{aligned}
\mid \mathcal{J}\left(u^{\varepsilon} ; \Omega(\varepsilon)\right)-\mathcal{J}(v ; \Omega(\varepsilon)) & -\int_{\Omega(\varepsilon)}\left(w\left(\varepsilon^{-1} x\right)+\varepsilon v^{\prime}(x)+\hat{u}^{\varepsilon}(x)\right) J_{v}^{\prime}(x, v(x)) \mathrm{d} x \mid \\
\leq & c \int_{\Omega(\varepsilon)}\left|w\left(\varepsilon^{-1} x\right)+\varepsilon v^{\prime}(x)+\hat{u}^{\varepsilon}(x)\right|^{1+\sigma} \mathrm{d} x \\
\leq & c \int_{\Omega(\varepsilon)}\left(\left|\frac{x}{\varepsilon}\right|^{-1-\sigma}+|x|^{-(1+\sigma)(\beta-2-\alpha)}\right. \\
& \cdot\left(\varepsilon^{1+\sigma}\left\|v^{\prime} ; \Lambda_{\beta}^{2, \alpha}(\Omega)\right\|^{1+\sigma}\right. \\
& \left.\left.+\left\|\hat{u}^{\varepsilon}(x) ; \Lambda_{\beta}^{2, \alpha}(\Omega)\right\|^{1+\sigma}\right)\right) \mathrm{d} x \\
\leq & c \varepsilon^{1+\sigma}\left(\int_{\varepsilon}^{1} r^{-1-\sigma} r^{2} \mathrm{~d} r\right. \\
& \left.+\int_{\varepsilon}^{1} r^{-(1+\sigma)(\beta-2-\alpha)} r^{2} \mathrm{~d} r\left(\varepsilon^{1+\sigma}+\varepsilon^{(1+\kappa)(1+\sigma}\right)\right) \\
\leq & c \varepsilon^{1+\sigma} .
\end{aligned}
$$


Here we have taken into account the fact that $1+\sigma \leq 2$, $(1+\sigma)(\beta-2-\alpha) \leq 2$, and both the integrals, extended on the interval $(0,1)$, do converge.

It suffices to mention the following inequalities:

$$
\begin{gathered}
\left|\mathcal{J}\left(u^{\varepsilon} ; \Omega(\varepsilon)\right)-\mathcal{J}(v ; \Omega)\right| \leq c \operatorname{mes}_{3}\left(\omega_{\varepsilon}\right) \leq c \varepsilon^{3}, \\
\int_{\Omega(\varepsilon)}\left|w\left(\varepsilon^{-1} x\right)+a \varepsilon \Phi(x)\right|\left|J_{v}^{\prime}(x, v(x))\right| \mathrm{d} x \\
\leq c \int_{\varepsilon}\left(\frac{r}{\varepsilon}\right)^{-2} r \mathrm{~d} r \leq c \varepsilon^{2}, \\
\int_{\Omega(\varepsilon)}\left|\hat{u}^{\varepsilon}\right|\left|J_{v}^{\prime}(x, v(x))\right| \mathrm{d} x \\
\leq c \varepsilon^{1+\kappa} \int_{\Omega(\varepsilon)}|x|^{-(\beta-2-\alpha)} \mathrm{d} x \leq c \varepsilon^{1+\kappa} .
\end{gathered}
$$

This confirms the formal calculations performed above. Let us formulate the main result in three dimensions.

Theorem 5. Under the assumptions listed above, we have

$$
\begin{array}{r}
\left|\mathcal{J}\left(u^{\varepsilon} ; \Omega(\varepsilon)\right)-\mathcal{J}(v ; \Omega)+\varepsilon 4 \pi v(\mathcal{O}) p(0) \operatorname{cap}(\omega)\right| \\
\leq c \varepsilon^{1+\min (\sigma, \kappa)} .
\end{array}
$$

\section{Topological derivative for a mixed semilinear elliptic problem in two spatial dimensions}

The numerical analysis is performed in two spatial dimensions. Therefore, we introduce a mixed semilinear problem and analyze the asymptotic in such a case.

Since the proof uses the same arguments as in three spatial dimensions (note that we use the Hölder norms, which are insensitive to the space dimension), we provide only the formal analysis and impose the Neumann boundary conditions on the hole boundary $\partial \omega_{\varepsilon}$. Note that the Dirichlet condition on $\partial \omega_{\varepsilon}$ changes crucially the form of asymptotic expansions cf. (Il'in, 1989; Mazja et al., 1981; Mazja et al., 1991).

3.1. Formal asymptotic analysis. Let $\Omega$ and $\omega$ be bounded domains in the plane $\mathbb{R}^{2}$. We consider the nonlinear mixed problem in the singularly perturbed domain $\Omega(\varepsilon)$, defined in (2):

$$
\left\{\begin{aligned}
-\Delta_{x} u^{\varepsilon}(x) & =F\left(x, u^{\varepsilon}(x)\right), & & x \in \Omega(\varepsilon), \\
u^{\varepsilon}(x) & =0, & & x \in \partial \Omega, \\
\partial_{n} u^{\varepsilon}(x) & =0, & & x \in \partial \omega_{\varepsilon} .
\end{aligned}\right.
$$

Referring to (Il'in, 1989) and especially to (Mazja et al., 1981; Mazja et al., 1991), we set

$$
\begin{aligned}
u^{\varepsilon}(x)= & v(x)+\varepsilon w_{1}\left(\varepsilon^{-1} x\right)+\varepsilon^{2} w_{2}\left(\varepsilon^{-1}(x)\right. \\
& +\varepsilon^{2} v^{\prime}(x)+\cdots,
\end{aligned}
$$

where $v, v^{\prime}$ and $w_{1}, w_{2}$ are components of regular and boundary layer types, respectively. Precisely, $v$ is a smooth solution of the problem (7) in the two dimensional entire domain $\Omega$. The Taylor formula yields

$v(x)=v(\mathcal{O})+x^{T} \nabla_{x} v(\mathcal{O})+\frac{1}{2} x^{T} \nabla_{x}^{2} v(\mathcal{O}) x+O\left(|x|^{3}\right)$.

The second term $w_{1}$ in the asymptotic ansatz (50) becomes a solution of the exterior problem

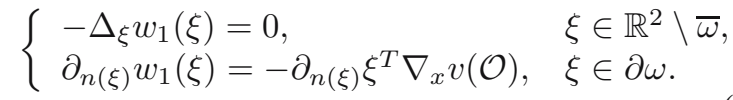

Such a solution admits the asymptotic representation

$w_{1}(\xi)=-\frac{1}{2 \pi} \frac{\xi^{T}}{|\xi|^{2}} m(\omega) \nabla_{x} v(\mathcal{O})+O\left(|\xi|^{-2}\right), \quad|\xi| \rightarrow \infty$,

where $m$ denotes the virtual mass matrix, see (Pólya and Szegö, 1951). Then the third term $w_{2}$ in (32) satisfies the problem

$$
\left\{\begin{aligned}
-\Delta_{\xi} w_{2}(\xi) & =0, & & \xi \in \mathbb{R}^{2} \backslash \bar{\omega} \\
\partial_{n(\xi)} w_{2}(\xi) & =-\partial_{n(\xi)} \frac{1}{2} \xi^{T} \nabla_{x} v(\mathcal{O}) \xi, & & \xi \in \partial \omega .
\end{aligned}\right.
$$

For such a solution, we write down the classical asymptotic representation

$$
w_{2}(\xi)=\frac{c}{2 \pi} \ln \frac{1}{|\xi|}+O\left(\frac{1}{|\xi|}\right), \quad|\xi| \rightarrow \infty,
$$

where the constant $c$ can be calculated as follows:

$$
\int_{\partial \omega} \partial_{n(\xi)} w_{2}(\xi) \mathrm{d} s_{\xi}=-\int_{\partial \mathbb{B}_{R}} \frac{\partial}{\partial|\xi|} \frac{c}{2 \pi} \ln \frac{1}{|\xi|} \mathrm{d} \xi=c .
$$

By the Green formula, we compute the left boundary integral

$$
\begin{aligned}
-\int_{\partial \omega} \partial_{n(\xi)} & \frac{1}{2} \xi^{T} \nabla_{x}^{2} v(\mathcal{O}) \xi \mathrm{d} s_{\xi} \\
& =\int_{\omega} \Delta_{\xi} \frac{1}{2} \xi^{T} \nabla_{x}^{2} v(\mathcal{O}) \xi \mathrm{d} \xi \\
& =\operatorname{mes}_{2} \omega \Delta_{x} v(\mathcal{O}) \\
& =-\operatorname{mes}_{2} \omega F(\mathcal{O} ; v(\mathcal{O})) .
\end{aligned}
$$

Finally, the fourth term $v^{\prime}$ in (32) is to be found from the Dirichlet problem

$$
\left\{\begin{aligned}
-\Delta_{x} v^{\prime}(x)= & \left(-\frac{1}{2 \pi} \frac{x^{T}}{|x|^{2}} m(\omega) \nabla_{x} v(\mathcal{O})\right. \\
& -\frac{1}{2 \pi} \ln \frac{\varepsilon}{|x|} \operatorname{mes}_{2} \omega F(\mathcal{O} ; v(\mathcal{O})) \\
& \left.+v^{\prime}(x)\right) F_{v}^{\prime}(x, v(x)), \quad x \in \Omega, \\
v^{\prime}(x)=\frac{1}{2 \pi} & \frac{x^{T}}{|x|^{2}} m(\omega) \nabla_{x} v(\mathcal{O}) \\
+ & \frac{1}{2 \pi} \ln \frac{\varepsilon}{|x|} \operatorname{mes}_{2} \omega F(\mathcal{O} ; v(\mathcal{O}), \quad x \in \partial \Omega .
\end{aligned}\right.
$$


3.2. Formal asymptotic of the shape functional. We introduce the following hypotheses:

(H7) $F \in C^{0, \alpha}(\bar{\Omega} \times \mathbb{R}), F_{v}^{\prime} \in C^{0, \alpha}(\bar{\Omega} \times \mathbb{R})$ for some $\alpha \in(0,1)$ and $F_{v}^{\prime} \leq 0$.

(H8) $J \in C^{0, \alpha}(\bar{\Omega} \times \mathbb{R}), J_{v}^{\prime} \in C^{0, \alpha}(\bar{\Omega} \times \mathbb{R})$

By the monotonicity of $F$, the Lax-Milgram lemma and the regularity of $J$, the problem

$$
\begin{cases}-\Delta_{x} p(x)-F_{v}^{\prime}(x, v(x)) p(x) & \\ =J_{v}^{\prime}(x, v(x)), & x \in \Omega, \\ p(x)=0, & x \in \partial \Omega\end{cases}
$$

admits a unique solution $p \in C^{2, \alpha}(\Omega)$.

We replace the solution $u^{\varepsilon}$ by its asymptotic representation (32). As a result, we obtain the first asymptotic term of order $\varepsilon^{2}$ for the shape functional

$$
\begin{aligned}
\mathcal{J} & \left(u^{\varepsilon} ; \Omega(\varepsilon)\right) \\
= & \int_{\Omega(\varepsilon)} J(x, v(x)) \mathrm{d} x+\int_{\Omega(\varepsilon)}\left(\varepsilon w_{1}\left(\varepsilon^{-1} x\right)\right. \\
& \left.+\varepsilon^{2} w_{2}\left(\varepsilon^{-1} x\right)+\varepsilon^{2} v^{\prime}(x)\right) J_{v}^{\prime}(x, v(x)) \mathrm{d} x+\cdots \\
= & \mathcal{J}(v ; \Omega(\varepsilon))+\varepsilon^{2} \int_{\Omega(\varepsilon)}\left(-\frac{1}{2 \pi} \frac{x^{T}}{|x|^{2}} m(\omega) \nabla_{x} v(\mathcal{O})\right. \\
& -\frac{1}{2 \pi} \ln \frac{\varepsilon}{|x|} \operatorname{mes}_{2} \omega F(\mathcal{O} ; v(\mathcal{O})) \\
& +v^{\prime}(x) J_{v}^{\prime}(x, v(x)) \mathrm{d} x+\cdots \\
= & \mathcal{J}(v ; \Omega)-\varepsilon^{2} \operatorname{mes}_{2} \omega J(\mathcal{O} ; v(\mathcal{O})) \\
& +\varepsilon^{2} \int\left(-\frac{1}{2 \pi} \frac{x^{T}}{|x|^{2}} m(\omega) \nabla_{x} v(\mathcal{O})\right. \\
& -\frac{1}{2 \pi} \ln \frac{\varepsilon}{|x|} \operatorname{mes}_{2} \omega F(\mathcal{O} ; v(\mathcal{O})) \\
& \left.+v^{\prime}(x)\right) J_{v}^{\prime}(x, v(x)) \mathrm{d} x+\cdots .
\end{aligned}
$$

Now we replace the right-hand side of (56) according to the equation and twice integrate by parts in the domain $\Omega \backslash \mathbb{B}_{\delta}=\{x \in \Omega:|x|>\delta\}$. We have

$$
\begin{aligned}
\int_{\Omega} & \left(-\frac{1}{2 \pi} \frac{x^{T}}{|x|^{2}} m(\omega) \nabla_{x} v(\mathcal{O})\right. \\
& \left.-\frac{1}{2 \pi} \ln \frac{\varepsilon}{|x|} \operatorname{mes}_{2} \omega F(\mathcal{O} ; v(\mathcal{O}))+v^{\prime}(x)\right) J_{v}^{\prime}(x, v(x)) \mathrm{d} x \\
= & -\lim _{\delta \rightarrow 0} \int_{\Omega \backslash \mathbb{B}_{\delta}}\left(\Delta_{x} p(x)+F_{v}^{\prime}(x, v(x)) p(x)\right) \\
& \cdot\left(-\frac{1}{2 \pi} \frac{x^{T}}{|x|^{2}} m(\omega) \nabla_{x} v(\mathcal{O})\right. \\
& \left.-\frac{1}{2 \pi} \ln \frac{\varepsilon}{|x|} \operatorname{mes}_{2} \omega F(\mathcal{O} ; v(\mathcal{O}))+v^{\prime}(x)\right) \mathrm{d} x
\end{aligned}
$$

$$
\begin{aligned}
= & -\lim _{\delta \rightarrow 0} \int_{\Omega \backslash \mathbb{B}_{\delta}} p(x)\left(\Delta_{x}+F_{v}^{\prime}(x, v(x))\right) \\
& \left(-\frac{1}{2 \pi} \frac{x^{T}}{|x|^{2}} m(\omega) \nabla_{x} v(\mathcal{O})-\frac{1}{2 \pi} \ln \frac{\varepsilon}{|x|} \operatorname{mes}_{2} \omega F(\mathcal{O} ; v(\mathcal{O}))\right. \\
& \left.+v^{\prime}(x)\right) \mathrm{d} x-\lim _{\delta \rightarrow 0} \int_{\partial \Omega} \partial_{n} p(x)\left(-\frac{1}{2 \pi} \frac{x^{T}}{|x|^{2}} m(\omega) \nabla_{x} v(\mathcal{O})\right. \\
& \left.-\frac{1}{2 \pi} \ln \frac{\varepsilon}{|x|} \operatorname{mes}_{2} \omega F(\mathcal{O} ; v(\mathcal{O}))+v^{\prime}(x)\right) \mathrm{d} x \\
& -\lim _{\delta \rightarrow 0} \int_{\partial \mathbb{B}_{\delta}}\left(\partial _ { | x | } p ( x ) \left(-\frac{1}{2 \pi} \frac{x^{T}}{|x|^{2}} m(\omega) \nabla_{x} v(\mathcal{O})\right.\right. \\
& \left.-\frac{1}{2 \pi} \ln \frac{\varepsilon}{|x|} \operatorname{mes}_{2} \omega F(\mathcal{O} ; v(\mathcal{O}))+v^{\prime}(x)\right) \\
& -p(x) \partial_{|x|}\left(-\frac{1}{2 \pi} \frac{x^{T}}{|x|^{2}} m(\omega) \nabla_{x} v(\mathcal{O})\right. \\
& \left.-\frac{1}{2 \pi} \ln \frac{\varepsilon}{|x|} \operatorname{mes}_{2} \omega F(\mathcal{O} ; v(\mathcal{O}))+v^{\prime}(x)\right) \mathrm{d} x .
\end{aligned}
$$

On the other hand, the boundary condition (55) implies that

$$
\begin{array}{r}
-\frac{1}{2 \pi} \frac{x^{T}}{|x|^{2}} m(\omega) \nabla_{x} v(\mathcal{O})-\frac{1}{2 \pi} \ln \frac{\varepsilon}{|x|} \operatorname{mes}_{2} \omega F(\mathcal{O} ; v(\mathcal{O})) \\
+v^{\prime}(x)=0 .
\end{array}
$$

Furthermore, for the linearized operator $\Delta_{x}+F_{v}^{\prime}$, the formula

$$
\begin{aligned}
\left(\Delta_{x}\right. & \left.+F_{v}^{\prime}(x, v(x))\right)+\left(-\frac{1}{2 \pi} \frac{x^{T}}{|x|^{2}} m(\omega) \nabla_{x} v(\mathcal{O})\right. \\
& \left.-\frac{1}{2 \pi} \ln \frac{\varepsilon}{|x|} \operatorname{mes}_{2} \omega F(\mathcal{O} ; v(\mathcal{O}))+v^{\prime}(x)\right) \\
= & \Delta_{x} v^{\prime}(x) \\
& +v^{\prime}(x) F_{v}^{\prime}(x, v(x)) \\
& +\left(-\frac{1}{2 \pi} \frac{x^{T}}{|x|^{2}} m(\omega) \nabla_{x} v(\mathcal{O})\right. \\
& \left.-\frac{1}{2 \pi} \ln \frac{\varepsilon}{|x|} \operatorname{mes}_{2} \omega F(\mathcal{O} ; v(\mathcal{O}))\right) F_{v}^{\prime}(x, v(x))=0
\end{aligned}
$$

is valid because the function

$$
\begin{aligned}
x \mapsto( & -\frac{1}{2 \pi} \frac{x^{T}}{|x|^{2}} m(\omega) \nabla_{x} v(\mathcal{O}) \\
& -\frac{1}{2 \pi} \ln \frac{\varepsilon}{|x|} \operatorname{mes}_{2} \omega F(\mathcal{O} ; v(\mathcal{O}))
\end{aligned}
$$

is a harmonics. Hence, we obtain that

$$
\begin{aligned}
\int_{\Omega}\left(-\frac{1}{2 \pi}\right. & \frac{x^{T}}{|x|^{2}} m(\omega) \nabla_{x} v(\mathcal{O}) \\
& \left.-\frac{1}{2 \pi} \ln \frac{\varepsilon}{|x|} \operatorname{mes}_{2} \omega F(\mathcal{O}) ; v(\mathcal{O})\right) \\
& \left.+v^{\prime}(x)\right) J_{v}^{\prime}(x, v(x)) \mathrm{d} x
\end{aligned}
$$




$$
\begin{aligned}
& -\lim _{\delta \rightarrow 0} \int_{\partial \mathbb{B}_{\delta}}\left(\partial _ { | x | } p ( x ) \left(-\frac{1}{2 \pi} \frac{x^{T}}{|x|^{2}} m(\omega) \nabla_{x} v(\mathcal{O})\right.\right. \\
& \left.-\frac{1}{2 \pi} \ln \frac{\varepsilon}{|x|} \operatorname{mes}_{2} \omega F(\mathcal{O} ; v(\mathcal{O}))\right) \\
& -p(x) \partial_{|x|}\left(-\frac{1}{2 \pi} \frac{x^{T}}{|x|^{2}} m(\omega) \nabla_{x} v(\mathcal{O})\right. \\
& \left.-\frac{1}{2 \pi} \ln \frac{\varepsilon}{|x|} \operatorname{mes}_{2} \omega F(\mathcal{O} ; v(\mathcal{O}))\right) \mathrm{d} x \\
& -\lim _{\delta \rightarrow 0} \sum_{i, j, k=1}^{2} \int_{\partial \mathbb{B}_{\delta}}\left[\frac{x_{i}}{|x|} \frac{\partial p}{\partial x_{i}}(\mathcal{O})\left(-\frac{1}{2 \pi} \frac{x_{k}}{|x|^{2}} m_{k j} \frac{\partial v}{\partial x_{j}}(\mathcal{O})\right)\right. \\
& \left.-\left(p(\mathcal{O})+x_{i} \frac{\partial p}{\partial x_{i}}(\mathcal{O})\right)\left(-\frac{1}{2 \pi} \frac{x_{j}}{|x|^{3}} m_{j k} \frac{\partial v}{\partial x_{k}}(\mathcal{O})\right)\right] \mathrm{d} x \\
& +\lim _{\delta \rightarrow 0} \int_{\partial \mathbb{B}_{\delta}}\left[\sum_{i=1}^{2}\left(p(\mathcal{O})+x_{i} \frac{\partial p}{\partial x_{i}}(\mathcal{O})\right)\right. \\
& \left.\cdot \times\left(\frac{1}{2 \pi} \frac{1}{|x|} \operatorname{mes}_{2} \omega F(\mathcal{O} ; v(\mathcal{O}))\right)\right] \mathrm{d} x \\
& -\lim _{\delta \rightarrow 0} \int_{\partial \mathbb{B}_{\delta}}\left[\sum_{i, j, k=1}^{2} \frac{x_{i}}{|x|} \frac{\partial p}{\partial x_{i}}(\mathcal{O})\left(-\frac{1}{\pi} \frac{x_{k}}{|x|^{2}} m_{k j} \frac{\partial v}{\partial x_{j}}(\mathcal{O})\right)\right. \\
& \left.-p(\mathcal{O})\left(\frac{1}{2 \pi} \frac{1}{|x|} \operatorname{mes}_{2} \omega F(\mathcal{O} ; v(\mathcal{O}))\right)\right] \mathrm{d} x \\
& \cdot \lim _{\delta \rightarrow 0} \int_{\partial \mathbb{B}_{\delta}}\left[\sum_{i, j=1}^{2} \frac{x_{i}}{|x|} \frac{\partial p}{\partial x_{i}}(\mathcal{O})\left(\frac{1}{\pi} \frac{x_{i}}{|x|^{2}} m_{i j} \frac{\partial v}{\partial x_{j}}(\mathcal{O})\right)\right. \\
& \left.+p(\mathcal{O})\left(\frac{1}{2 \pi} \frac{1}{|x|} \operatorname{mes}_{2} \omega F(\mathcal{O} ; v(\mathcal{O}))\right)\right] \mathrm{d} x \\
& =F(\mathcal{O} ; v(\mathcal{O})) \operatorname{mes}_{2} \omega p(\mathcal{O}) \\
& +\nabla_{x} p(\mathcal{O})^{T} m(\omega) \nabla_{x} v(\mathcal{O}) \text {. }
\end{aligned}
$$

Thus, recalling (57) we conclude the relation

$$
\begin{aligned}
\mathcal{J} & \left(u^{\varepsilon} ; \Omega(\varepsilon)\right) \\
= & \mathcal{J}(v ; \Omega)+\varepsilon^{2}\left[-\operatorname{mes}_{2} \omega J(\mathcal{O} ; v(\mathcal{O}))\right. \\
& +F(\mathcal{O} ; v(\mathcal{O})) \operatorname{mes}_{2} \omega p(\mathcal{O}) \\
& \left.+\nabla_{x} p(\mathcal{O})^{T} m(\omega) \nabla_{x} v(\mathcal{O})\right]+\cdots
\end{aligned}
$$

Theorem 6. Under the assumptions (H1), (H7) and (H8), the asymptotic expansion (58) is valid with the remainder $o\left(\varepsilon^{2}\right)$.

\section{Finite element approximations of topological derivatives}

Our aim in this section is to compute a numerical approximation of the topological derivative of the shape functional (8), with $u^{\varepsilon}$ being the solution of the problem (49), and give $L^{\infty}$-estimates of the error.
4.1. Family of finite elements. In $\bar{\Omega}$ we consider a family of triangulations $\left\{\mathscr{T}_{h}\right\}_{h>0}$. With each element $T \in$ $\mathscr{T}_{h}$, we associate two parameters $\rho(T)$ and $\sigma(T)$, where $\rho(T)$ denotes the diameter of the set $T$, and $\sigma(T)$ is the diameter of the largest ball contained in $T$. We set $h=$ $\max _{T \in \mathscr{T}_{h}} \rho(T)$. We make the following assumptions on the triangulations:

(H10) Regularity assumption: There exists $\sigma>0$ such that $\rho(T) / \sigma(T) \leq \sigma$ for $T \in \mathscr{T}_{h}$ and $h>0$.

(H11) Inverse assumption: There exists $\rho>0$ such that $h / \rho(T) \leq \rho$ for $T \in \mathscr{T}_{h}$ and $h>0$.

(H12) We denote by $\bar{\Omega}_{h}=\cup_{T \in \mathscr{T}_{h}} T$ the domain obtained by a triangulation, with $\Omega_{h}$ as its interior and $\partial \Omega_{h}$ its boundary. Then we assume that the vertices of $\mathscr{T}_{h}$ placed on the boundary $\partial \Omega_{h}$ also belong to $\partial \Omega$.

Consider the spaces

$$
\begin{array}{r}
V_{h}=\left\{v_{h} \in C(\bar{\Omega}):\left.v_{h}\right|_{T} \in P_{1}(T) \text { for } T \in \mathscr{T}_{h}\right. \\
\text { and } \left.v_{h}=0 \text { in } \Omega \backslash \Omega_{h}\right\}
\end{array}
$$

and

$$
W_{h}=\left\{v_{h} \in C\left(\bar{\Omega}_{h}\right):\left.v_{h}\right|_{T} \in P_{1}(T) \text { for } T \in \mathscr{T}_{h}\right\},
$$

where $P_{1}(T)$ is the space of polynomials of degree 1 on $T, V_{h}$ is a vector subspace of $H_{0}^{1}(\Omega)$ and $W_{h}$ is a subspace of $H^{1}(\Omega)$.

We use the Lagrange interpolation operator

$$
\Pi_{h}: C(\bar{\Omega}) \rightarrow W_{h}
$$

$\Pi_{h} z$ being the unique element in $W_{h}$ such that $\Pi_{h} z\left(x_{i}\right)=$ $z\left(x_{i}\right)$ for every node $x_{i}$ of the triangulation. In the case of a function $z$ vanishing on $\partial \Omega$, we extended $\Pi_{h} z$ to $\bar{\Omega}$ by zero and we denote this extension by $\Pi_{h} z$, too. In the last case, we have that $\Pi_{h} z \in V_{h}$.

4.2. Numerical solution of the semilinear problem. By virtue of the assumption $F \in C^{0,1}(\bar{\Omega} \times \mathbb{R})$ and by the mean value theorem, we deduce the following local Lipschitz condition: For all $M>0$ there exists $c_{M}>0$ such that

$$
\begin{array}{r}
\left|F\left(x, v_{1}\right)-F\left(x, v_{2}\right)\right| \leq c_{M}\left|v_{1}-v_{2}\right|, \quad x \in \Omega, \\
\left|v_{1}\right|,\left|v_{2}\right| \leq M .
\end{array}
$$

Using classical arguments, we can deduce from the monotonicity of $F(x, \cdot)$ and 59 ) the existence of a unique solution to $(7)$ in $H_{0}^{1}(\Omega) \cap C(\bar{\Omega})$. We refer to (Stampacchia, $1965)$ for the boundedness of the solution. From to the convexity of $\Omega$, we can deduce that the solution is in $H^{2}(\Omega)$ (Ladyzhenskaya and Ural'tseva, 1968).

The weak formulation of the equation (7) is the following:

$$
a(v, z)=(F(x, v), z)_{L^{2}(\Omega)}, \quad z \in H_{0}^{1}(\Omega),
$$


where

$$
a(v, z)=\int_{\Omega} \nabla v(x) \nabla z(x) \mathrm{d} x .
$$

The numerical approximation $v_{h}$ of $v$ is then the solution of the problem

$$
\left\{\begin{array}{l}
\text { Find } v_{h} \in V_{h} \text { such that } \\
a\left(v_{h}, z_{h}\right)=\left(F\left(x, v_{h}\right), z_{h}\right)_{L^{2}(\Omega)}, \text { for any } z_{h} \in V_{h} .
\end{array}\right.
$$

The proof of the existence of a solution of 610 is well known (Stampacchia, 1965). It is enough to apply, in a convenient way, Browder's fixed point theorem along with the monotonicity of $F(x, \cdot)$.

We rewrite problem (56) in the form

$$
\left\{\begin{aligned}
-\Delta p(x) & =F_{0}(x, p(x)), & & x \in \Omega, \\
p(x) & =0, & & x \in \partial \Omega,
\end{aligned}\right.
$$

where $F_{0}(x, p(x))=F_{v}^{\prime}(x, v(x)) p(x)+J^{\prime}(x, v(x)) . F_{0}$ is linear with respect to the second variable, so that $F_{0} \in$ $C^{0,1}(\bar{\Omega} \times \mathbb{R})$.

Then the variational formulation of the linear problem 56 is the following:

$$
a(p, z)=\left(F_{0}(x, p), z\right)_{L^{2}(\Omega)}, \quad z \in H_{0}^{1}(\Omega) .
$$

Thus, numerical approximation of $p$ is the solution of the variational problem

$$
\left\{\begin{array}{l}
\text { Find } p_{h} \in V_{h} \text { such that } \\
a\left(p_{h}, z_{h}\right)=\left(F_{0}\left(x, p_{h}\right), z_{h}\right)_{L^{2}(\Omega)} \text { for any } z_{h} \in V_{h} .
\end{array}\right.
$$

Due to the hypotheses (H1) and (H7), the linear problem (64) has a solution in space $V_{h}$.

In the next section we give $L^{\infty}$-estimates for the finite element approximation of $v, \nabla v, p, \nabla p$ and the topological derivatives in the case of the semilinear elliptic problem.

\subsection{Convergence of finite element approximation for} the semilinear problem and the adjoint state problem. We are going to use the recent results on the convergence of the finite element method in $W^{1, \infty}$ spaces. The topological derivative is a pointwise expression with the values of the solution and the adjoint state, as well as with the values of the gradient of these functions. In order to derive the error estimates in the $L^{\infty}$ norm for the topological derivative, it is required to have in hand the error estimates in the $W^{1, \infty}$ norm for the solutions of the semilinear equation as well as the linear adjoint state equation. The results presented below lead to the error estimate for the topological derivative. We refer the reader to (Casas and Mateos, 2002; Demlov, 2007; Frehse and Rannacher, 1978), for the proofs of error estimates for linear and semilinear elliptic equations.

The following $L^{\infty}$-estimate for approximation by finite elements of solutions to the problem (7) was proved in (Casas and Mateos, 2002).
Theorem 7. Let $v$ and $v_{h}$ be solutions of the variational problems (60) and (61), respectively. Then there exists a constant $C>0$ independent of $h$ such that

$$
\left\|v-v_{h} ; L^{\infty}\left(\Omega_{h}\right)\right\| \leq C h\left\|v ; H^{2}(\Omega)\right\| .
$$

In addition, assuming that $\Omega_{h} \subset \Omega$, we have the following estimates, proved in (Demlov, 2007) (see also (Frehse and Rannacher, 1978)).

Theorem 8. Let $v$ and $v_{h}$ be solutions of the variational problems (60) and (61), respectively. Then there exists a constant $C>0$ independent of $h$ such that

$$
\left\|\nabla v-\nabla v_{h} ; L^{\infty}\left(\Omega_{h}\right)\right\|_{\infty} \leq C h\left\|v ; H^{2}(\Omega)\right\| .
$$

On the other hand, we have the following $L^{\infty}$ - estimates for the approximation of solutions to the linear problem (56).

Theorem 9. Let $x_{0} \in \Omega$, and let $p$ and $p_{h}$ be solutions of the variational problems (63) and (64), respectively. Then there exists a constant $C>0$ independent of $h$ such that

$$
\begin{aligned}
\left\|p-p_{h} ; L^{\infty}\left(\Omega_{h}\right)\right\|+\| \nabla p & -\nabla p_{h} ; L^{\infty}\left(\Omega_{h}\right) \| \\
& \leq C h\left\|p ; H^{2}(\Omega)\right\|
\end{aligned}
$$

4.4. $L^{\infty}$ - estimates for the approximation of the topological derivative. We denote by $\mathcal{T}_{\Omega, h}$ numerical approximation by the finite element method of the topological derivative $\mathcal{T}_{\Omega}$. Then

$$
\begin{aligned}
\mathcal{T}_{\Omega, h}(\mathcal{O})= & -\operatorname{mes}_{2}(\omega) J\left(\mathcal{O}, v_{h}(\mathcal{O})\right) \\
& +\nabla_{x} p_{h}(\mathcal{O})^{T} m(\omega) \nabla_{x} v_{h}(\mathcal{O}) \\
& +F\left(\mathcal{O}, v_{h}(\mathcal{O})\right) \operatorname{mes}_{2}(\omega) p_{h}(\mathcal{O}) .
\end{aligned}
$$

We obtain

$$
\begin{aligned}
\mid \mathcal{T}_{\Omega}(\mathcal{O})- & \mathcal{T}_{\Omega, h}(\mathcal{O}) \mid \\
= & \mid-\operatorname{mes}_{2}(\omega)\left[J(\mathcal{O}, v(\mathcal{O}))-J\left(\mathcal{O}, v_{h}(\mathcal{O})\right)\right] \\
& +\left[\nabla_{x} p(\mathcal{O})^{T} m(\omega) \nabla_{x} v(\mathcal{O})\right. \\
& \left.-\nabla_{x} p_{h}(\mathcal{O})^{T} m(\omega) \nabla_{x} v_{h}(\mathcal{O})\right] \\
& +\left[F(\mathcal{O}, v(\mathcal{O})) \operatorname{mes}_{2}(\omega) p(\mathcal{O})\right. \\
& \left.-F\left(\mathcal{O}, v_{h}(\mathcal{O})\right) \operatorname{mes}_{2}(\omega) p_{h}(\mathcal{O})\right] \mid \\
\leq & \operatorname{mes}_{2}(\omega)\left|J(\mathcal{O}, v(\mathcal{O}))-J\left(\mathcal{O}, v_{h}(\mathcal{O})\right)\right| \\
& +\mid \nabla_{x} p(\mathcal{O})^{T} m(\omega) \nabla_{x} v(\mathcal{O}) \\
& -\nabla_{x} p_{h}(\mathcal{O})^{T} m(\omega) \nabla_{x} v_{h}(\mathcal{O}) \mid \\
& +\operatorname{mes}_{2}(\omega) \mid F(\mathcal{O}, v(\mathcal{O})) p(\mathcal{O}) \\
& -F\left(\mathcal{O}, v_{h}(\mathcal{O})\right) p_{h}(\mathcal{O}) \mid
\end{aligned}
$$

We have

$$
\begin{aligned}
&\left|J(\mathcal{O}, v(\mathcal{O}))-J\left(\mathcal{O}, v_{h}(\mathcal{O})\right)\right| \\
& \leq c\left\|v-v_{h} ; L^{\infty}\left(\Omega_{h}\right)\right\|
\end{aligned}
$$


It follows that

$$
\begin{aligned}
& \left|\mathcal{T}_{\Omega}(\mathcal{O})-\mathcal{T}_{\Omega, h}(\mathcal{O})\right| \\
& \leq c\left\|v-v_{h} ; L^{\infty}\left(\Omega_{h}\right)\right\| \\
& \quad+\left|\nabla_{x} p(\mathcal{O})^{T} m(\omega) \nabla_{x}\left(v(\mathcal{O})-v_{h}(\mathcal{O})\right)\right| \\
& \quad+\left|\nabla_{x}\left(p(\mathcal{O})-p_{h}(\mathcal{O})\right)^{T} m(\omega) \nabla_{x} v_{h}(\mathcal{O})\right| \\
& \quad+\operatorname{mes}_{2}(\omega)\left|F(\mathcal{O}, v(\mathcal{O}))\left(p(\mathcal{O})-p_{h}(\mathcal{O})\right)\right| \\
& \quad+\operatorname{mes}_{2}(\omega)\left|\left(F(\mathcal{O}, v(\mathcal{O}))-F\left(\mathcal{O}, v_{h}(\mathcal{O})\right)\right) p_{h}(\mathcal{O})\right| .
\end{aligned}
$$

Therefore

$$
\begin{array}{r}
\left|F(\mathcal{O}, v(\mathcal{O}))-F\left(\mathcal{O}, v_{h}(\mathcal{O})\right)\right| \\
\leq c\left\|v-v_{h} ; L^{\infty}\left(\Omega_{h}\right)\right\|,
\end{array}
$$

and we obtain

$$
\begin{aligned}
& \left|\mathcal{T}_{\Omega}(\mathcal{O})-\mathcal{T}_{\Omega, h}(\mathcal{O})\right| \\
& \leq c\left\|v-v_{h} ; L^{\infty}\left(\Omega_{h}\right)\right\| \\
& \quad+c\left\|\nabla_{x}\left(v(\mathcal{O})-v_{h}(\mathcal{O})\right) ; L^{\infty}\left(\Omega_{h}\right)\right\| \\
& \quad+c\left\|\nabla_{x}\left(p(\mathcal{O})-p_{h}(\mathcal{O})\right) ; L^{\infty}\left(\Omega_{h}\right)\right\| \\
& \quad+c\left\|p(\mathcal{O})-p_{h}(\mathcal{O}) ; L^{\infty}\left(\Omega_{h}\right)\right\| .
\end{aligned}
$$

Finally, by Theorems 8 and 9 , we deduce the following result.

Theorem 10. The following error estimate holds for the evaluation of the topological derivatives:

$$
\begin{aligned}
& \left|\mathcal{T}_{\Omega}(\mathcal{O})-\mathcal{T}_{\Omega, h}(\mathcal{O})\right| \\
& \quad \leq C h\left(\left\|v ; H^{2}(\Omega)\right\|+\left\|p ; H^{2}(\Omega)\right\|\right) .
\end{aligned}
$$

4.5. Numerical examples. In this section, we present some numerical examples to show the behavior of topological derivative approximation with respect to the evolution of discretization step size. We derive errors and verify that the computed error satisfies the estimate from Theorem (10) in each case.

For each of the examples, we choose the domain $\Omega$ as a square $(0,1) \times(0,1)$ and the following energy functional:

$$
\begin{aligned}
\mathcal{J}(v ; \Omega)= & \frac{1}{2} \int_{\Omega}|\nabla v(x)|^{2} \mathrm{~d} x+\frac{1}{4} \int_{\Omega} v^{4}(x) \mathrm{d} x \\
& -\int_{\Omega} f(x) v(x) \mathrm{d} x
\end{aligned}
$$

where $v$ is the solution of the nonlinear problem

$$
\left\{\begin{aligned}
-\Delta_{x} v(x) & =-v(x)^{3}+f(x), & & x \in \Omega, \\
v(x) & =0, & & x \in \partial \Omega .
\end{aligned}\right.
$$

We take different right-hand sides $f$ in each of the examples. The size of discretization is determined by $h$, which decreases in each iteration. We compute the error in 20 iterations starting with $h=0.2$ and reduce it by 0.01 in each step.
Example 1. In the first example the function $f$ is given by

$$
\begin{aligned}
f(x)= & f\left(x_{1}, x_{2}\right)=\left(\left(x_{1}^{2}+x_{2}^{2}\right) \sin \pi x_{1} \sin \pi x_{2}\right)^{3} \\
& +2\left(\pi^{2}\left(x_{1}^{2}+x_{2}^{2}\right)-2\right) \sin \pi x_{1} \sin \pi x_{2} \\
& -4 \pi\left(x_{1} \cos \pi x_{1} \sin \pi x_{2}+\sin \pi x_{1} \cos \pi x_{2}\right) .
\end{aligned}
$$

We calculate the exact solution of 73

$$
u\left(x_{1}, x_{2}\right)=\left(x_{1}^{2}+x_{2}^{2}\right) \sin \pi x_{1} \sin \pi x_{2},
$$

and the corresponding adjoint state

$$
p\left(x_{1}, x_{2}\right)=-\frac{1}{2}\left(x_{1}^{2}+x_{2}^{2}\right) \sin \pi x_{1} \sin \pi x_{2} .
$$

Exact and numerical approximations of the topological derivative are presented in Fig. 1. In Fig. 2 we plot the relative evolution of the error and the behavior of the error with respect to the discretization step size.
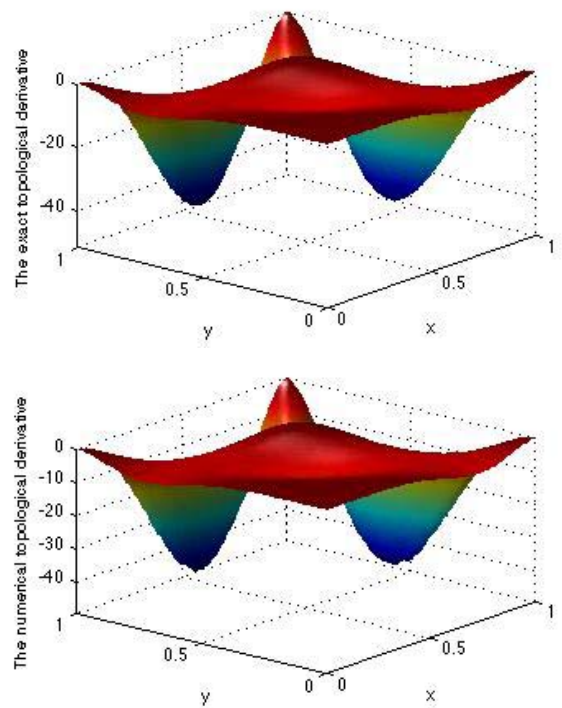

Fig. 1. Topological derivative: exact $\mathcal{T}_{\Omega}$ (left) and approximate $\mathcal{T}_{\Omega, h}$ (right).

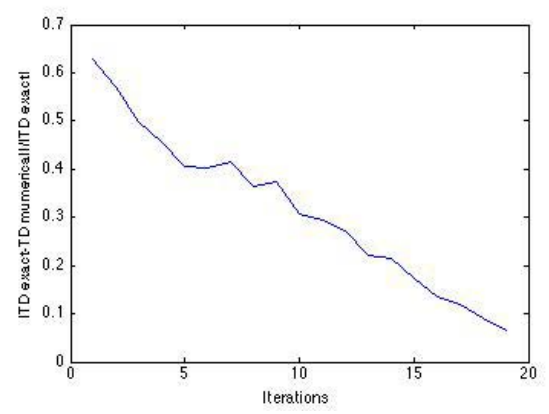

Fig. 2. Error : $\left|\mathcal{T}_{\Omega}-\mathcal{T}_{\Omega, h}\right|_{\infty} /\left|\mathcal{T}_{\Omega}\right|_{\infty}$. 
Example 2. Let us choose

$$
\begin{aligned}
f(x)= & f\left(x_{1}, x_{2}\right) \\
= & \left(x_{1}\left(x_{1}-1\right) x_{2}\left(x_{2}-1\right)\right)^{3} \\
& -2\left(x_{2}\left(x_{2}-1\right)+x_{1}\left(x_{1}-1\right)\right) .
\end{aligned}
$$

In this case,

$$
u\left(x_{1}, x_{2}\right)=x_{1}\left(x_{1}-1\right) x_{2}\left(x_{2}-1\right)
$$

and

$$
p\left(x_{1}, x_{2}\right)=-\frac{1}{2} x_{1}\left(x_{1}-1\right) x_{2}\left(x_{2}-1\right) .
$$

The exact topological derivative and it numerical approximation are presented in Fig. 3 and in Fig. 4 we show the relative evolution of the error and the behavior of the error with respect to the discretization step size.
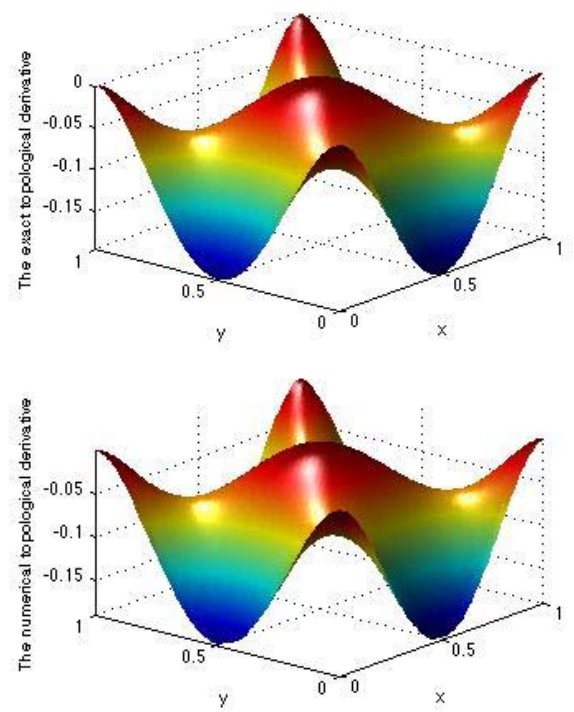

Fig. 3. Topological derivative: exact $\mathcal{T}_{\Omega}$ (left) and approximate $\mathcal{T}_{\Omega, h}$ (right).

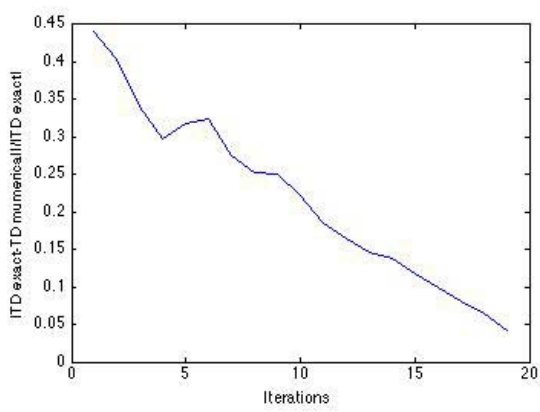

Fig. 4. Error : $\left|\mathcal{T}_{\Omega}-\mathcal{T}_{\Omega, h}\right|_{\infty} /\left|\mathcal{T}_{\Omega}\right|_{\infty}$.
Example 3. In the last example, we take

$$
\begin{aligned}
f\left(x_{1}, x_{2}\right)= & \left(100 x_{1}^{2} x_{2}^{2}\left(x_{1}-1\right)^{2}\left(x_{2}-1\right)^{2}\right)^{3} \\
& -200\left[x_{1}^{2}\left(x_{1}-1\right)^{2}\left(6 x_{2}^{2}-6 x_{2}+1\right)\right. \\
& \left.+x_{2}^{2}\left(x_{2}-1\right)^{2}\left(6 x_{1}^{2}-6 x_{1}+1\right)\right] .
\end{aligned}
$$

Then

$$
u\left(x_{1}, x_{2}\right)=100 x_{1}^{2} x_{2}^{2}\left(x_{1}-1\right)^{2}\left(x_{2}-1\right)^{2}
$$

and

$$
p\left(x_{1}, x_{2}\right)=-50 x_{1}^{2} x_{2}^{2}\left(x_{1}-1\right)^{2}\left(x_{2}-1\right)^{2} .
$$

For this last example the exact topological derivative and it numerical approximation are presented in Fig. 5, and in Fig. 6 we show the relative evolution of the error and the behavior of the error with respect to the discretization step size.

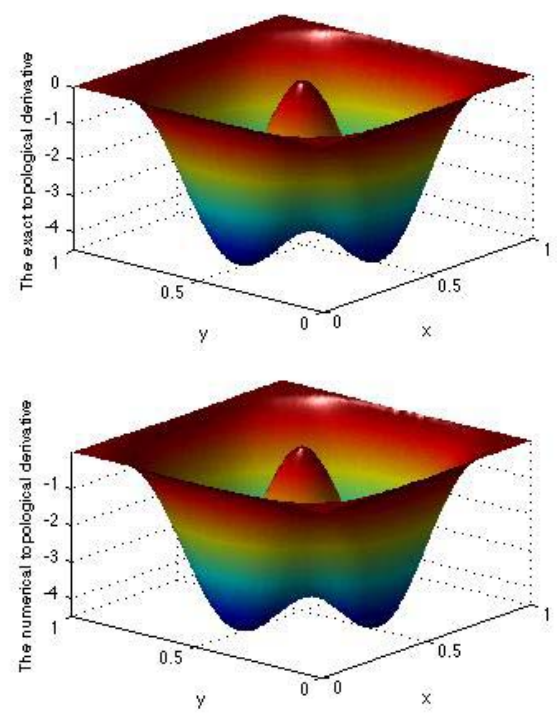

Fig. 5. Topological derivative: exact $\mathcal{T}_{\Omega}$ (left) and approximate $\mathcal{T}_{\Omega, h}$ (right).

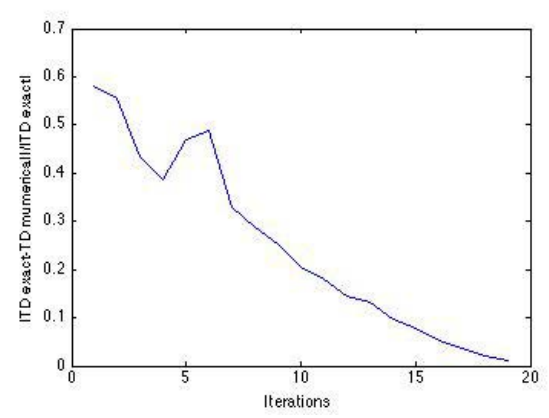

Fig. 6. Error : $\left|\mathcal{T}_{\Omega}-\mathcal{T}_{\Omega, h}\right|_{\infty} /\left|\mathcal{T}_{\Omega}\right|_{\infty}$. 


\section{Conclusions}

In the paper the form of topological derivatives of the integral shape functional was obtained for semilinear elliptic boundary value problems in two and three spatial dimensions. The finite element method was used to compute an approximation of the topological derivatives. Convergence analysis of the finite element method was performed in two spatial dimensions. Since the application of topological derivatives in shape optimization requires pointwise values, we provided $L^{\infty}$-estimates for finite element approximations. The results of computations confirmed the a priori estimate of the numerical approximation error. The presented results can be used for shape and topology optimization for semilinear elliptic boundary value problems.

\section{References}

Amstutz, S. (2006). Topological sensitivity analysis for some nonlinear PDE system, Journal Mathematiques Pures et Appliquées 85(4): 540-557.

Bucur, D. and Buttazzo, G. (2005). Variational Methods in Shape Optimization Problems, Birkhäuser, Boston, MA.

Casas, E. and Mateos, M. (2002). Uniform convergence of the FEM. Applications to state constrained control problems, Journal of Computational and Applied Mathematics 21(1): $67-100$.

Ciarlet, P. G. (1978). The Finite Element Method for Elliptic Problems, North-Holland, Amsterdam.

Ciarlet, P. G. and Raviart, P. A. (1972). General Lagrange and Hermite interpolation in $\mathbb{R}^{n}$ with applications to finite element methods, Archive for Rational Mechanics and Analysis 46(3): 177-199.

Demlov, A. (2007). Sharply localized pointwise and $W_{\infty}^{-1}$ estimates for finite element methods for quasilinear problems, Mathematics of Computation 76(260): 1725-1741.

Frehse, J. and Rannacher, R. (1978). Asymptotic $L^{\infty}$-error estimates for linear finite element approximations of quasilinear boundary value problems, SIAM Journal on Numerical Analysis 15(2): 418-431.

Fulmanski, P., Lauraine, A., Scheid, J.-F. and Sokołowski, J. (2007). A level set method in shape and topology optimization for variational inequalities, International Journal of Applied Mathematics and Computer Science 17(3): 413430.

Gilbarg, D. and Trudinger, N. S. (2001). Elliptic Partial Differential Equations of Second Order, Springer-Verlag, Berlin.

Haug, E. J. and Céa, J. (1981). Optimization of distributed parameter structures, Proceedings of the NATO Advanced Study Institute on Optimization of Distributed Parameter Structural Systems, Iowa City, IO, USA, NATO Advanced Study Institute Series E: Applied Sciences, Vol. 49, Martinus Nijhoff Publishers, The Hague.

Il'in, A. M. (1989). Matching of Asymptotic Expansions of Solutions of Boundary Value Problems, Nauka, Moscow, (in Russian).
Jackowska-Strumillo, L., Sokołowski, J., Zochowski, A. and Henrot, A. (2002). On numerical solution of shape inverse problems, Computational Optimization and Applications 23(2): 231-255.

Kondratiev, V. A. (1967). Boundary problems for elliptic equations in domains with conical or angular points, Trudy Moskovskogo Matematicheskogo Obszhestva 16: 209-292, (in Russian).

Ladyzhenskaya, O. A. and Ural'tseva, N. N. (1968). Linear and Quasilinear Elliptic Equations, Academic Press, New York, NY London.

Landkof, N. S. (1966). Fundamentals of Modern Potential Theory, Nauka, Moscow, (in Russian).

Mazja, V. G., Nazarov, S. A. and Plamenevskii, B. A. (1981). On the asymptotic behavior of solutions of elliptic boundary value problems with irregular perturbations of the domain, Problemy Matematicheskogo Analiza 8: 72-153.

Mazja, V. G., Nazarov, S. A. and Plamenevskii, B. A. (1991) Asymptotische Theorie elliptischer Randwertaufgaben in singulär gestörten, Gebieten. Bd. 1, Akademie-Verlag, Berlin; English translation: Asymptotic Theory of Elliptic Boundary Value Problems in Singularly Perturbed Domains, Vol. 1, Birkäuser Verlag, Basel, 2000.

Mazja, V. G. and Plamenevskii, B. A. (1978). Estimates in $L_{p}$ and Hölder classes and the Miranda-Agmon maximum principle for solutions of elliptic boundary value problems in domains with singular points on the boundary, Matematische Nachrichten 81(1): 25-82, (in Russian).

Mazja, V. G. and Plamenevskii, B. A. (1973). On the behavior of solutions to quasilinear elliptic boundary-value problems in a neighborhood of a conical point, Zapiski Nauchnych Seminarov Leningradskogo Otdeleniya Matematicheskogo Instituta (LOMI) 38: 91-97.

Nazarov, S. A. and Plamenevsky, B. A. (1973). Elliptic Problems in Domains with Piecewise Smooth Boundaries, Walter de Gruyter, Berlin.

Nazarov, S. A. and Sokolowski, J. (2006). Self-adjoint extensions for the Neumann Laplace and applications, Acta Mathematica Sinica 22(3): 879-906.

Nazarov, S. A. and Sokolowski, J. (2003). Asymptotic analysis of shape functional, Journal de Mathématiques Pures et Appliquées 82(2): 125-196.

Pólya, G. P. and Szegö, G. (1951). Isoperimetric Inequalities in Mathematical Physics, Princeton University Press, Princeton, $\mathrm{NJ}$.

Raviart, P. A. and Thomas, J. M. (1983). Introduction à l'analyse numérique des équations aux dérives partielles Masson, Paris.

Sokolowski, J. and Zochowski, A. (1999). Asymptotic analysis of shape functional, Numerische Mathematik 102(1): 145179.

Sokolowski, J. and Zochowski, A. (2005). Introduction to Shape Optimization. Shape Sensitivity Analysis, Springer-Verlag, Berlin. 
Sokolowski, J. and Zochowski, A. (1999). On topological derivative in shape optimization, SIAM Journal on Control and Optimization 37(4): 1251-1272.

Sokolowski, J. and Zochowski, A. (2003). Optimality conditions for simultaneous topology and shape optimization, SIAM Journal on Control and Optimization 42(4): 1198-1221.

Sokolowski, J. and Zochowski, A. (2001). Topological derivatives of shape functional for elasticity systems, Mechanics of Structures and Machines 29(3): 331-349.

Sokolowski, J. and Zochowski, A. (1999). On topological derivative in shape optimization, SIAM Journal on Control and Optimization 37(4): 1251-1272.

Stampacchia, G. (1965). Le problème de Dirichlet pour les équations elliptiques du second ordre à coefficients discontinus, Annales de I'Institut Fourier 15: 189-258.

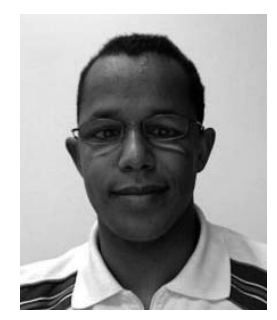

Mohamed Iguernane

2007-ongoing: assistant professor, King Khalid University, Abha, Saudi Arabia.

2005-2007: temporary teaching and research position, Nancy University, France.

2006: university research post-doctoral diploma, Nancy University, France.

2004-2005: post-doctoral position, Elie Cartan Institute, Nancy, France.

2003-2004: teaching fellow, Cadi Ayyad University, Marrakesh, Morocco.

2002: Ph.D. in applied mathematics, Cadi Ayyad University, Marrakesh, Morocco.

1997: Master's degree in dynamical systems, Cadi Ayyad University, Marrakesh, Morocco.

Research interests: partial differential equations, numerical analysis, optimization, bifurcation, topological theory, statistics.

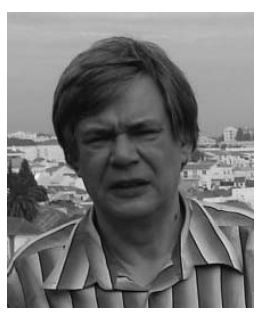

Serguei A. Nazarov is a professor at the Institute of Mechanical Engineering Problems in St. Petersburg, an author of 14 research monographs and about 400 research papers. He specializes in qualitative analysis of partial differential equations and variational inequalities. His research is focused on asymptotic analysis of solutions of elliptic boundary value problems in domains with irregular boundaries and/or singular perturbations. The results are used in fracture theory, elasticity and hydro-dynamics, the theory of waves, and shape optimization.

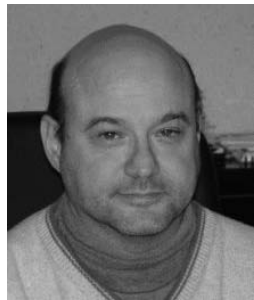

Jean-Rodolphe Roche received a Ph.D. degree in mathematics in 1980 from the Joseph Fourier University of Grenoble and a habilitation qualification in mathematics in 1996 from the Henry Poincaré University of Nancy. At present he is a professor of numerical analysis at Henry Poincaré University. His research interest are shape optimization, domain decomposition for non-linear problems, and semiLagrangian methods in plasma physics.

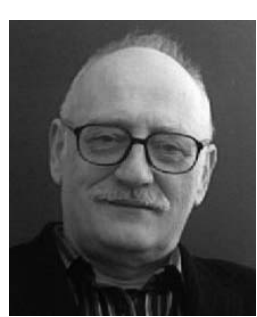

Jan Sokolowski is a professor of applied mathematics at the Elie Cartan Institute of Henry Poincaré University in Nancy, an author of two research monographs (with Jean-Paul Zolesio and Alexander Khludnev) and 150 research papers on the modelling and optimization of distributed parameter systems described by partial differential equations of solid and fluid mechanics. His recent research fields include asymptotic analysis and topological derivatives in shape optimization (with Antoni Zochowski and Sergey Nazarov), and the modelling and shape optimization of compressible Navier-Stokes equations (with Pavel I. Plotnikov).

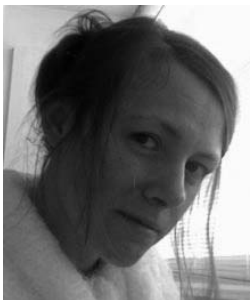

\section{Katarzyna Szulc}

Since 2005: Ph.D. student, Henry Poincaré University of Nancy, France.

Research interests: partial differential equations, numerical analysis, shape optimization.

Received: 28 April 2008 Revised: 27 October 2008 